\title{
Novel Conformationally Locked Inositols: From Aromatics to Annulated Cyclitols
}

\author{
Goverdhan Mehta,* Ramesh S. Senaiar, and Mrinal K. Bera ${ }^{[a]}$
}

\begin{abstract}
A new family of ring-annulated inositols with "locked" conformations has been designed to deliver a range of these biologically important entities in "unnatural conformations" while retaining their "natural configurations". The simple "tool" of trans ring fusion has been used to "lock" the conformation of the annulated inositols. Short, simple syntheses of a range of
\end{abstract}

these novel cyclitols have been achieved from readily available aromatic precursors such as tetralin and indane. Along the way, annulated $C_{2}$-symmetric cyclohexadiene-trans-diol (trans-CHD) de-

Keywords: annulation - conformation analysis - cyclitols • dihydroxylation $\cdot$ signal transduction rivatives have been prepared for the first time and serve as the pivotal building blocks for generating the oxy-functionalization pattern of inositols. The presence of chemo-differentiated hydroxyl groups in our novel inositols is expected to facilitate the installation of phosphate diversity to harness the biological potential of these entities.

\section{Introduction}

Inositols 1 occupy a preeminent position among biologically important entities known as cyclitols and constitute the only group of cyclohexanes substituted at each carbon atom for which all the possible (nine) diastereomers, five natural (myo, scyllo, D-chiro, L-chiro, and neo) and four synthetic (cis, epi, allo, and muco), are known. ${ }^{[1]}$ The biological functions of derivatives of inositol are wide-ranging and diverse and include intercellular communication, phosphate storage and transfer, anti-cancer and involvement in covalent anchoring of proteins to membranes. ${ }^{[1 \mathrm{a}-\mathrm{c}, 2]}$ However, it is the pivotal role of inositol phosphate derivatives such as D-myo-inositol-1,4,5triphosphate $\left[\operatorname{Ins}(1,4,5) \mathrm{P}_{3}\right]$ and D-myo-inositol-1,3,4,5-tetrakisphosphate $\left[\operatorname{Ins}(1,3,4,5) \mathrm{P}_{4}\right]$ as second messengers in intercellular signal transduction events through binding to specific receptors and mobilizing $\mathrm{Ca}^{2+}$ ions from intracellular stores that has generated contemporary interest in their chemistry and biology. ${ }^{[1,2]}$ The increased cytosolic $\mathrm{Ca}^{2+}$ concentration initiates a number of cell-type specific responses. ${ }^{[1 \mathrm{~b}, 2]}$ Thus, intervention and selective manipulation of physiological processes triggered by inositol polyphosphates has stimulated the search for new and designer analogues for unraveling the complex biological mechanisms and development of new pharmaceuticals.

[a] Prof. G. Mehta, R. S. Senaiar, M. K. Bera

Department of Organic Chemistry

Indian Institute Science

Bangalore 560012 (India)

Fax: (+91) 80-360-0936

E-mail: gm@orgchem.iisc.ernet.in
The quest for new synthetic analogues of inositols, though extensive, ${ }^{[3,4]}$ has largely centered on ring modification and sidearm and phosphate variation and studies with these structural variants continue to provide insights into structure-activity relationships. Here, we introduce a new family of ring-annulated bicyclic inositols 2 as novel entities with several unique attributes. The trans-fused bicyclic inositols $\mathbf{2}$, unlike $\mathbf{1}$, are rigid and can be locked in high-energy conformations (see below) which are unattainable in $\mathbf{1}^{\left[{ }^{[5]}\right.}$ For example, while the most abundant myo-inositol exists in the stable conformation $\mathbf{3}$ with five equatorial and one axial (5e/1a) hydroxyl group, the 1,6-annulated bicyclic myoinositol 4 would be locked in the five axial and one equatorial (5a/1e) conformation. Secondly, the presence of two chemodifferentiated tertiary hydroxyl groups in $\mathbf{2}$ is expected to facilitate the generation of functional group diversity and phosphate variation, an essential but complicated feature of inositol chemistry. ${ }^{[1]}$ It should be noted that selective phosphorylation of inositols is a cumbersome, multistep protocol which requires extensive protection-deprotection maneuvers. Lastly, a hydrophobic appendage in the form of an alicyclic ring in $\mathbf{2}$ could profoundly modulate the cell membrane permeability and receptor recognition parameters of the inositol moiety. Interestingly, the ring annulation maneuver on inositols, which leads to $\mathbf{2}$, can be expected to alter their reactivity and biological profile and has not been attempted before. ${ }^{[3 \mathrm{~h}]}$ More importantly, the annulated inositols are destined to be locked in "unnatural conformations" while retaining their "natural configurations". In this paper, we describe a simple and versatile approach to several cyclohexa- and cyclopenta-annulated inositols $\mathbf{2}$ from readily 


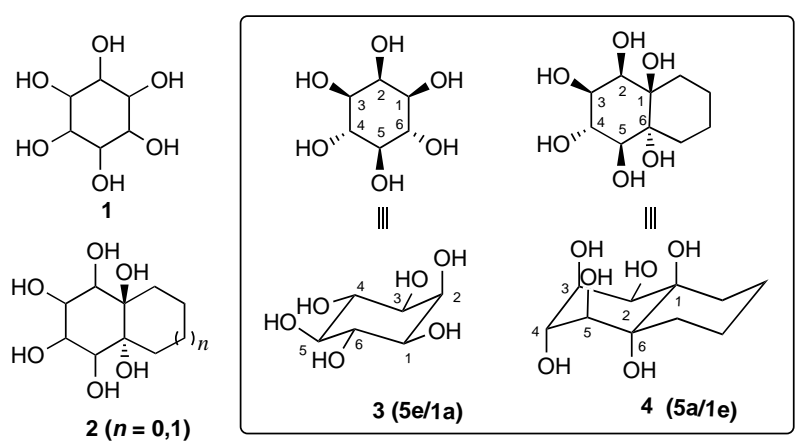

available aromatic precursors such as tetralin and indane. While the syntheses reported here are of racemic compounds, our overall strategy is amenable to chiral induction at several stages along the way.

In our general approach to 2 , annulated $C_{2}$-symmetric cyclohexadiene-trans-diol (trans-CHD) derivatives 5 and $\mathbf{6}$ were recognized as the pivotal building blocks and access to them from readily available precursors was first devised. ${ }^{[6,7]}$ For the synthesis of cyclohexa-annulated trans-CHD 5, 1,4dihydrotetralin (7) obtained from tetralin was subjected to regioselective epoxidation to give $\mathbf{8}$ followed by acid-catalyzed ring opening to a trans diol $^{[8]}$ and acetylation to furnish the trans diacetate 9 (Scheme 1). Allylic bromination of 9 and DBU (1,8-diazabicyclo[5.4.0]undec-7-ene)-mediated dehydrobromination delivered the desired $C_{2}$-symmetric cyclohexadiene-trans-diol diacetate 5. ${ }^{\left[{ }^{9]}\right.}$ In a similar manner, cyclopenta-annulated cyclohexadiene-trans-diol derivative 6 was prepared from 1,4-dihydroindane (10) via trans-diol 11 (Scheme 2). ${ }^{[10]}$ The allylic bromination-dehydrobromination sequence, successful in the case of $\mathbf{9}$, led only to intractable products when implemented on $\mathbf{1 1}$ or its diacetate derivative. After some trials, a convenient route to the $C_{2}$-symmetric trans-CHD derivative $\mathbf{6}$ from $\mathbf{1 1}$ was established through addition of bromine and acetylation to $\mathbf{1 2}$ and double dehydrobromination in the presence of DBU. ${ }^{[9]}$

The $C_{2}$-symmetric bicyclic trans-CHD diacetates 5 and 6 served as the key starting materials for accessing the new family of inositols. The main theme in our approach was to generate the network of oxygen functionalities on the diene moiety in a stereoselective manner and with the minimum number of steps. Mono-epoxidation of $\mathbf{5}$ was stereoselective and furnished a readily separable mixture of diastereomeric epoxides $\mathbf{1 3}$ and $\mathbf{1 4}(9: 1)$ (Scheme 1). Acid-catalyzed ring opening of the major epoxide 13 furnished bicyclic diols $15(3: 1)$ and 16, which are annulated conduritol $\mathrm{F}$ and $\mathrm{B}$ derivatives, respectively (Scheme 3). ${ }^{[11,12]}$ The stereostructure of the acetate migration product $\mathbf{1 5}$, though indicated through careful scrutiny of $6 \mathrm{~h}, 80 \%$.

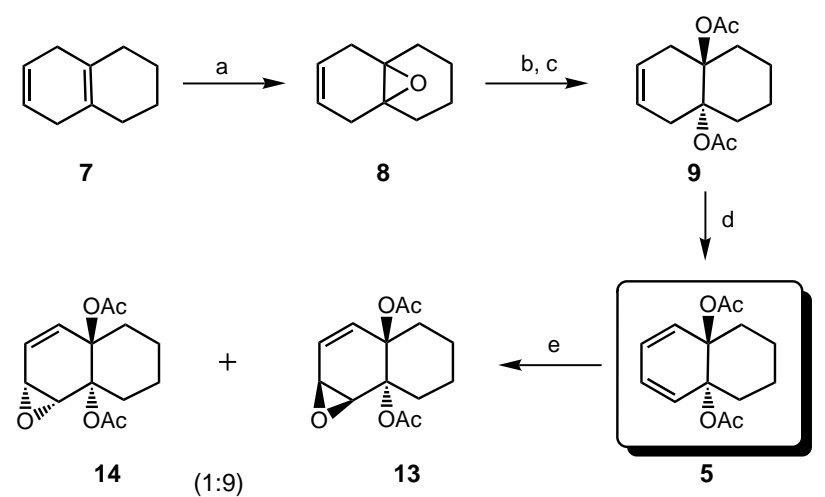

Scheme 1. a) $m \mathrm{CPBA}, \mathrm{CH}_{2} \mathrm{Cl}_{2},-5^{\circ} \mathrm{C}, 5 \mathrm{~min}, 85 \%$; b) $10 \% \mathrm{AcOH}$, RT, $2 \mathrm{~h}, 90 \%$; c) $\mathrm{Ac}_{2} \mathrm{O}, \mathrm{BF}_{3} \cdot \mathrm{Et}_{2} \mathrm{O}, \mathrm{RT}, 2 \mathrm{~h}, 88 \%$; d) i) NBS, AIBN, $\mathrm{CCl}_{4}$, reflux, $4 \mathrm{~h}$; ii) DBU, DMSO, RT, $52 \%$ (2 steps); e) $m \mathrm{CPBA} \mathrm{CH}_{2} \mathrm{Cl}_{2}, 10^{\circ} \mathrm{C}, 5-6 \mathrm{~h}$, $73 \%$ (based on recovered starting material).

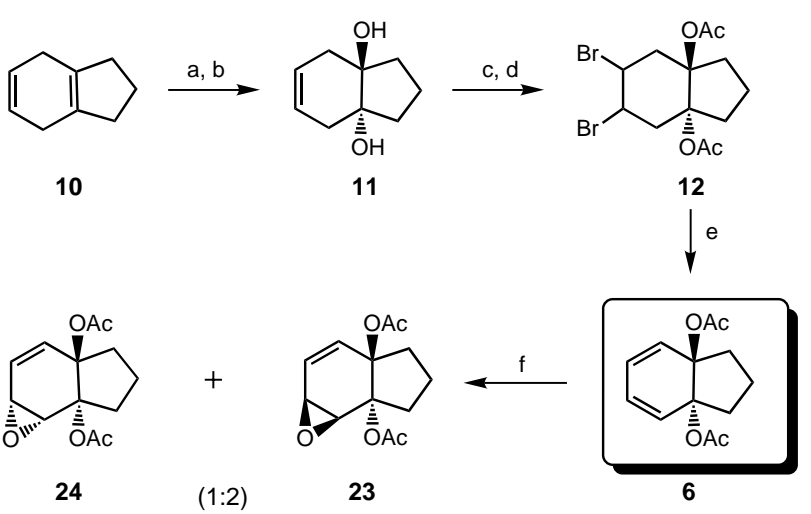

Scheme 2. a) MMPP (magnesium monoperoxyphthalate hexahydrate), $\mathrm{THF} / \mathrm{H}_{2} \mathrm{O} 1: 1, \quad 0^{\circ} \mathrm{C}, 5 \mathrm{~min}, 85 \%$; b) $10 \% \mathrm{AcOH}, \mathrm{RT}, 5 \mathrm{~h}, 90 \%$; c) $\mathrm{C}_{6} \mathrm{H}_{5} \mathrm{~N}^{+} \cdot \mathrm{HBr}_{3}^{-}, \mathrm{CH}_{2} \mathrm{Cl}_{2}, 0^{\circ} \mathrm{C}, 1 \mathrm{~h}, 66 \%$; d) $\mathrm{Ac}_{2} \mathrm{O}, \mathrm{BF}_{3} \cdot \mathrm{Et}_{2} \mathrm{O}, \mathrm{RT}, 1 \mathrm{~h}$, $89 \%$; e) DBU, DMSO, RT, 4 h, $54 \%$; f) $m \mathrm{CPBA}, \mathrm{CH}_{2} \mathrm{Cl}_{2}, 0{ }^{\circ} \mathrm{C}, 3 \mathrm{~d}, 60 \%$.

the spectral data, was secured through X-ray crystallography studies. The origin of $\mathbf{1 5}$ from epoxide $\mathbf{1 3}$ can be traced to an intramolecular neighboring acetate-mediated opening of the epoxide ring followed by an $\mathrm{S}_{\mathrm{N}} 2^{\prime}$ displacement by the distal acetate group as shown in Scheme 4. It is this eventful participation of both the acetate groups of $\mathbf{1 3}$ in the epoxide ring opening that generates the interesting cis-1,4-diol stereo-

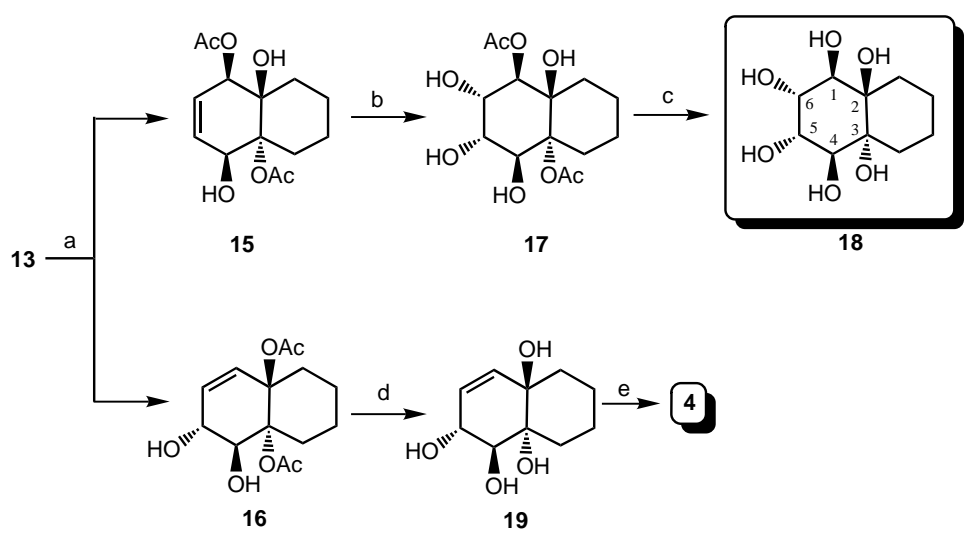

Scheme 3. a) $10 \% \mathrm{AcOH}, \mathrm{THF}, 50{ }^{\circ} \mathrm{C}, 16 \mathrm{~h}, 80 \%$; b) $\mathrm{OsO}_{4}$ (cat.), NMMO, acetone/water 4:1, RT, $2 \mathrm{~h}, 88 \%$; c) $\mathrm{K}_{2} \mathrm{CO}_{3}, \mathrm{MeOH}, \mathrm{RT}, 2 \mathrm{~h}, 96 \%$; d) $\mathrm{K}_{2} \mathrm{CO}_{3}, \mathrm{MeOH}, \mathrm{RT}, 1 \mathrm{~h}, 95 \%$; e) $\mathrm{OsO}_{4}$ (cat.), NMMO, acetone/water 4:1, RT, 


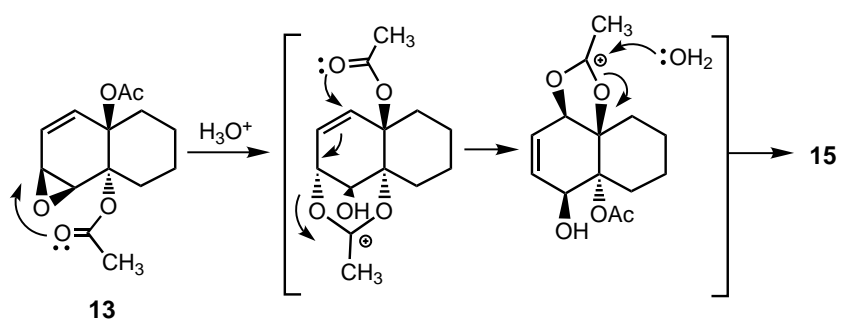

Scheme 4. Postulated mechanism of formation of $\mathbf{1 5}$.

chemistry observed in $\mathbf{1 5}$ (Scheme 4). ${ }^{[13]}$ The major product $\mathbf{1 5}$ from epoxide 13 was then subjected to stereospecific $\mathrm{OsO}_{4}$ mediated dihydroxylation to furnish the inositol derivative $\mathbf{1 7}$ (Scheme 3). The stereochemical outcome of the dihydroxylation of $\mathbf{1 5}$ to give $\mathbf{1 7}$ was predictable and expected both on steric considerations and Kishi's rule ${ }^{[14]}$ for the $\mathrm{OsO}_{4}$-mediated dihydroxylation of allylic alcohols. Base hydrolysis of $\mathbf{1 7}$ led to the 2,3-cyclohexa-annulated chiro-inositol 18. ${ }^{[15]}$ The most stable conformation of chiro-inositol determined by $\mathrm{X}$-ray crystallography is $4 \mathrm{e} / 2 \mathrm{a}$ as shown in Scheme 5. ${ }^{[16]}$ In contrast, the X-ray crystal structure of 2,3-cyclohexa-annulated chiro-inositol $\mathbf{1 8}$ exhibited a $4 \mathrm{a} / 2 \mathrm{e}$ conformation and is displayed for comparison in Scheme 5.

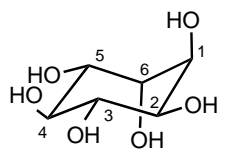

chiro-Inositol (4e/2a)

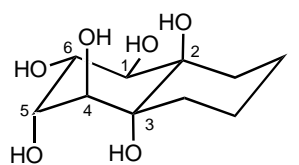

$18(4 a / 2 e)$
Scheme 5. Stable conformations of chiro-inositol and annulated chiroinositol 18.
Attention was now turned to the minor diol 16 obtained from the cleavage of epoxide $\mathbf{1 3}$. Acetate hydrolysis of $\mathbf{1 6}$ led to the tetrol 19, which is an annulated conduritol $\mathrm{B}$ derivative (Scheme 3). ${ }^{[11]}$ Catalytic $\mathrm{OsO}_{4}{ }^{-}$ mediated dihydroxylation of $\mathbf{1 9}$ was smooth and stereospecific and furnished the cyclohexaannulated myo-inositol 4. An X-ray crystal structure of $\mathbf{4}$ indicated that as per our design strategy it indeed had a $5 \mathrm{a} / 1 \mathrm{e}$ ground state conformation in contrast with $5 \mathrm{e} / 1 \mathrm{a}$ for myoinositol 3. ${ }^{[17]}$ Once again, dihydroxylation of $\mathbf{1 9}$ occurred from the face opposite to the secondary allylic hydroxyl group. ${ }^{[14]}$

In an alternate sequence, bicyclic diene diacetate $\mathbf{5}$ was subjected to exhaustive dihydroxylation with catalytic $\mathrm{OsO}_{4}$ to stereospecifically furnish the hexaoxygenated products $20(2: 1)$ and 21, with the latter arising through 1,4-acetate migration (Scheme 6). Base hydrolysis of both $\mathbf{2 0}$ and 21 furnished 3,4-cyclohexa-annulated chiro-inositol 22. ${ }^{[15]}$ The stereostructure of $\mathbf{2 2}$ was evident from the $C_{2}$ symmetry present and this annulated chiroinositol was locked in the $4 \mathrm{a} / 2 \mathrm{e}$ conformation. It is noteworthy that starting from the same trans-CHD diacetate 5, two inositols (18 and 22) with the chiro-configuration and 4a/2e conformation can be crafted by a tactic that is formally equivalent to altering the site of annulation (2,3- and 3,4-, respectively) ${ }^{[15]}$ on chiro-inositol.

The elaboration of the cyclohexadiene-trans-diol derivative 6 to diverse cyclopenta-annulated inositols followed the protocols successfully implemented on $\mathbf{5}$ (see above). Epoxidation of $\mathbf{6}$ was less stereoselective (cf. 5) and furnished a readily separable mixture (2:1) of epoxides $\mathbf{2 3}$ and $\mathbf{2 4}$ (Scheme 2). Acid-catalyzed ring opening of the major epoxide $\mathbf{2 3}$ furnished diols 25 and $\mathbf{2 6}$ in a 3:1 ratio (Scheme 7). The stereostructures of conduritol $F \mathbf{2 5}$ and conduritol B 26 derivatives ${ }^{[11]}$ were secured through $\mathrm{X}$-ray crystal structure determination. The major product was once again derived through complex acetate migration analogous to that observed for 13 (Scheme 4). ${ }^{[13]}$ Acetate hydrolysis in 25 furnished the annulated conduritol $\mathrm{F}$ derivative 27, and further catalytic $\mathrm{OsO}_{4}$-mediated dihydroxylation led stereospecifically to the 2,3-cyclopenta-annulated ${ }^{[15]}$ chiro-inositol 28. Inositol 28 was firmly locked in $4 \mathrm{a} / 2 \mathrm{e}$ conformation. Similarly, the minor epoxide cleavage product $\mathbf{2 6}$ was hydrolyzed to give conduritol B derivative 29, and further dihydroxylation furnished the cyclopenta-annulated myo-inositol 30 which was locked in the 5a/1e ground state conformation (Scheme 7). ${ }^{[17]}$ On acid cleavage the remaining minor epoxide $\mathbf{2 4}$ obtained from 6 furnished a single product $\mathbf{3 1}$ in which both the acetate

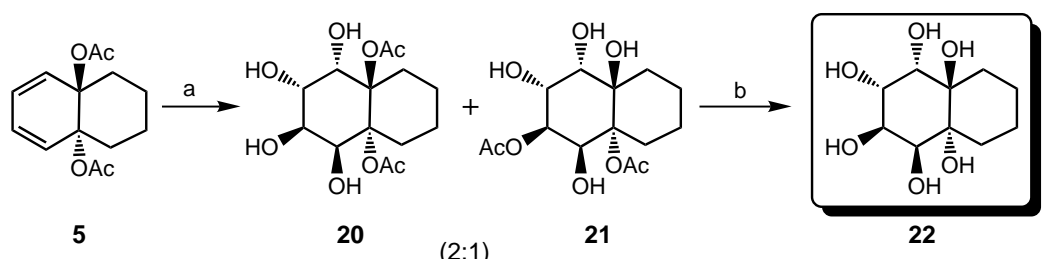

Scheme 6. a) $\mathrm{OsO}_{4}$ (cat.), NMMO, acetone/water 4:1, $10{ }^{\circ} \mathrm{C}, 2 \mathrm{~d}, 70 \%$; b) $\mathrm{K}_{2} \mathrm{CO}_{3}, \mathrm{MeOH}, \mathrm{RT}, 2 \mathrm{~h}, 90 \%$.

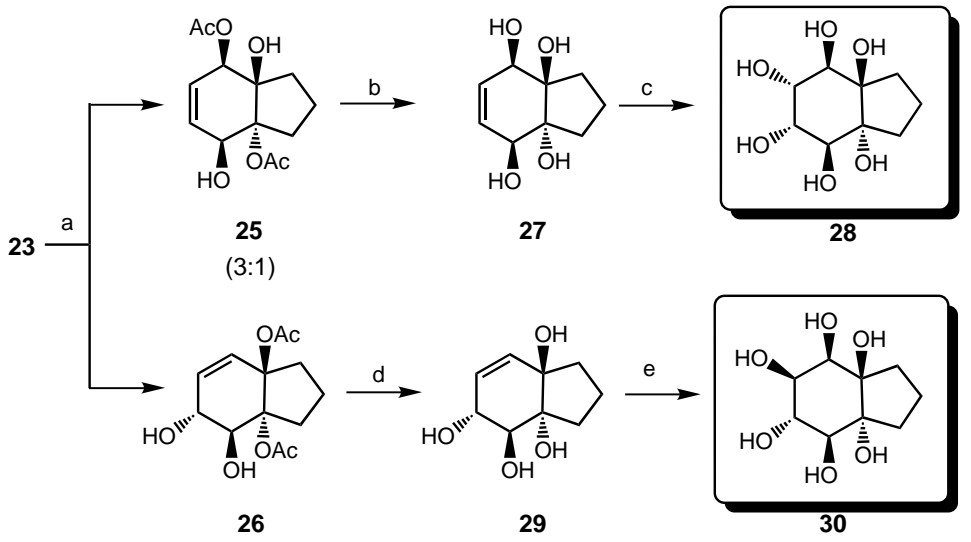

Scheme 7. a) $10 \% \mathrm{AcOH}$, THF, $50{ }^{\circ} \mathrm{C}, 24 \mathrm{~h}, 60 \%$; b) $\mathrm{K}_{2} \mathrm{CO}_{3}, \mathrm{MeOH}, \mathrm{RT}, 6 \mathrm{~h}, 81 \%$; c) $\mathrm{OsO}_{4}$ (cat.), NMMO, acetone/water 4:1, RT, 4 h, $84 \%$; d) $\mathrm{K}_{2} \mathrm{CO}_{3}$, $\mathrm{MeOH}, \mathrm{RT}, 4 \mathrm{~h}, 92 \%$; e) $\mathrm{OsO}_{4}$ (cat.), NMMO, acetone/water 4:1, RT, $3 \mathrm{~h}, 86 \%$. 


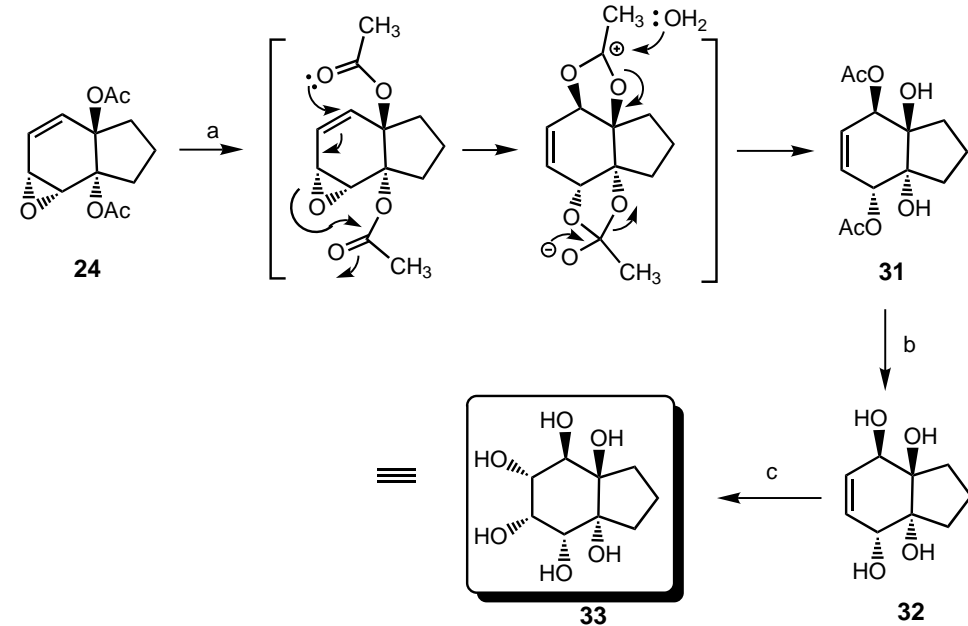

Scheme 8. a) $10 \% \mathrm{AcOH}, \mathrm{THF}, 50^{\circ} \mathrm{C}, 24 \mathrm{~h}, 74 \%$; b) $\mathrm{K}_{2} \mathrm{CO}_{3}, \mathrm{MeOH}, \mathrm{RT}, 3 \mathrm{~h}, 95 \%$; c) $\mathrm{OsO}_{4}$ (cat.), NMMO, acetone/water $4: 1$, RT, $3 \mathrm{~h}, 83 \%$.

groups had migrated (Scheme 8). Hydrolysis of $\mathbf{3 1}$ led to the conduritol $\mathrm{E}$ derivative 32, and further $\mathrm{OsO}_{4}$-mediated dihydroxylation furnished the annulated allo-inositol 33. The stereostructure of $\mathbf{3 3}$ was secured through single-crystal X-ray structure determination and had a locked $3 \mathrm{a} / 3 \mathrm{e}$ conformation.

Lastly, the annulated trans-CHD diol $\mathbf{6}$ was subjected to mono-dihydroxylation to stereospecifically give the diol $\mathbf{3 4}$ with concomitant 1,4-acetate migration (Scheme 9). The stereostructure of $\mathbf{3 4}$ was again secured through X-ray crystal structure determination. Acetate hydrolysis in $\mathbf{3 4}$ provided a conduritol $\mathrm{F}$ derivative $\mathbf{3 5}$, and a second $\mathrm{OsO}_{4}$-mediated dihydroxylation led to the 3,4-cyclopenta-annulated chiroinositol 36, ${ }^{[15]}$ which adopts a $4 \mathrm{a} / 2 \mathrm{e}$ conformation.

Experimental Section

General methods: Melting points were recorded on a Büchi B-540 apparatus and are uncorrected. Infrared spectra were recorded on JASCO FTIR 410. Proton ( $\left.{ }^{1} \mathrm{H} \mathrm{NMR}\right)$ and carbon magnetic resonance $\left({ }^{13} \mathrm{C}\right.$ NMR) spectra were generally recorded on a JEOL JNM-LA 300 spectrometer. Mass spectra measurements were carried out on a JEOL JMS DX 303 spectrometer. Elemental analyses were carried out on a Carlo Erba Element Analyzer 1106. Analytical thin-layer chromatography (TLC) was performed on $(10 \times 5 \mathrm{~cm})$ glass plates coated $(250 \mu \mathrm{m})$ with Acme silica gel $\mathrm{G}$ or $\mathrm{GF}_{254}$ (containing $13 \%$ calcium sulfate as binder). Visualization of the spots on TLC plates was achieved either by exposure to iodine vapor or UV light or by spraying sulfuric acid and heating the plates at $120^{\circ} \mathrm{C}$. Column chromatography was performed by using Acme silica gel $(100-200 \mathrm{mesh})$ or neutral alumina. All solvents were freshly distilled over $\mathrm{CaH}_{2}$ or $\mathrm{Na}$ /benzophenone as appropriate.

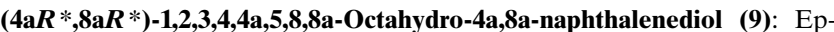
oxide $8(3 \mathrm{~g}, 0.02 \mathrm{~mol})^{[8]}$ was treated with $10 \%$ acetic acid $(10 \mathrm{~mL})$, and the resulting biphasic reaction mixture was stirred vigorously at room temperature. Upon complete consumption of the starting material (monitored by TLC), the acid was neutralized by careful addition of solid $\mathrm{NaHCO}_{3}$, and the resulting diol was extracted with ethyl acetate $(3 \times$ $100 \mathrm{~mL})$. The combined organic extracts were washed with saturated aqueous $\mathrm{NaHCO}_{3}(2 \times 50 \mathrm{~mL})$, brine $(1 \times 50 \mathrm{~mL})$, dried over anhydrous $\mathrm{Na}_{2} \mathrm{SO}_{4}$, and concentrated under reduced pressure to furnish the trans diol $(3 \mathrm{~g}, 90 \%)$. The diol was dissolved in acetic anhydride $(10 \mathrm{~mL})$ and a catalytic amount of $\mathrm{BF}_{3} \cdot \mathrm{Et}_{2} \mathrm{O}(100 \mu \mathrm{L})$ was added at $0{ }^{\circ} \mathrm{C}$. The resulting dark brown solution was stirred for $2 \mathrm{~h}$ under $\mathrm{N}_{2}$ atmosphere. The reaction mixture was poured into ice-cold water $(10 \mathrm{~mL})$ and extracted with $\mathrm{CH}_{2} \mathrm{Cl}_{2}$ $(3 \times 100 \mathrm{~mL})$. The combined organic extracts were washed with saturated aqueous $\mathrm{NaHCO}_{3}(2 \times 50 \mathrm{~mL})$, brine $(20 \mathrm{~mL})$, dried over anhydrous $\mathrm{Na}_{2}$. $\mathrm{SO}_{4}$, and concentrated under reduced pressure to furnish crystalline trans diacetate 9 ( $4.7 \mathrm{~g})$, which was recrystallized from hexane/dichloromethane to give colorless needle-like crystals of 9 (4.54 g, $88 \%$ ). M.p. $136-137^{\circ} \mathrm{C}$; IR $(\mathrm{KBr}): \quad \tilde{v}=1727 \mathrm{~cm}^{-1} ; \quad{ }^{1} \mathrm{H} \quad \mathrm{NMR}$ $\left(300 \mathrm{MHz}, \mathrm{CDCl}_{3}\right): \delta=5.15(\mathrm{~m}, 2 \mathrm{H})$, 3.09 (s, 1 H), 3.03 (s, 1 H), 2.69 (s, 1 H), $2.65(\mathrm{~s}, 1 \mathrm{H}), 2.36(\mathrm{~s}, 1 \mathrm{H}), 2.18(\mathrm{~s}, 1 \mathrm{H})$, $2.01(\mathrm{~s}, 6 \mathrm{H}), 1.66-1.13(\mathrm{~m}, 6 \mathrm{H})$; ${ }^{13} \mathrm{C}$ NMR $\left(75 \mathrm{MHz}^{1} \mathrm{CDCl}_{3}\right): \delta=169.6$

\section{Conclusion}

We have delineated short and efficient routes to ringannulated $C_{2}$-symmetric trans-CHD diols 5 and $\mathbf{6}$ and harnessed them to craft a range of novel bicyclic inositols. Our design of these unusual entities employs the annulation stratagem for generating new "unnatural" conformations and structural diversity while retaining the "natural" configurations of the inositols. The availability of such bicyclic inositols with differentiated hydroxyl groups with new spatial orientations augurs well for further phosphate variations and biological evaluation.
(2C), 123.2 (2C), 80.8 (2C), 29.9 (2C), 28.5 (2C), 22.3 (2C), 20.8 (2C); MS (70 eV, EI): $\mathrm{m} / z(\%): 252\left[\mathrm{M}^{+}\right]$; elemental analysis calcd (\%) for $\mathrm{C}_{14} \mathrm{H}_{20} \mathrm{O}_{4}$ (252): C 66.64, H 7.99; found: C 66.56, H 8.03.

$(4 \mathrm{a} R *, 8 \mathrm{a} R *)-8 \mathrm{a}-($ Acetyloxy)-1,2,3,4,4a,8a-hexahydro-4-naphthalenyl acetate (5): $N$-Bromosuccinimide $(706 \mathrm{mg}, 3.97 \mathrm{mmol})$ and a catalytic amount of 2,2'-azabisisobutyronitrile (AIBN) $(10 \mathrm{mg}$ ) were added to a solution of the trans-diacetate $9(1 \mathrm{~g}, 3.97 \mathrm{mmol})$ in $\operatorname{dry~}_{\mathrm{CCl}_{4}}(10 \mathrm{~mL})$, and the resulting reaction mixture was heated at reflux for $4 \mathrm{~h}$ under $\mathrm{N}_{2}$. After the completion of the reaction, floating succinimide was filtered off and the filtrate was poured into ice-cold water $(15 \mathrm{~mL})$. The organic layer was separated and aqueous layer was extracted with $\mathrm{CH}_{2} \mathrm{Cl}_{2}(3 \times 20 \mathrm{~mL})$. The combined organic extracts were successively washed with $10 \% \mathrm{HCl},(2 \times$ $10 \mathrm{~mL})$, water $(10 \mathrm{~mL})$, brine $(10 \mathrm{~mL})$, dried over anhydrous $\mathrm{Na}_{2} \mathrm{SO}_{4}$, and concentrated under reduced pressure. The residue $(1.3 \mathrm{~g})$ obtained was dissolved in dry DMSO $(10 \mathrm{~mL})$ and DBU $(0.7 \mathrm{~mL}, 4.76 \mathrm{mmol})$ was added 
gradually from a syringe at $5-10^{\circ} \mathrm{C}$ under $\mathrm{N}_{2}$ atmosphere, during which time the reaction mixture turned from pale yellow to dark brown. The reaction mixture was stirred at room temperature for further $2 \mathrm{~h}$, before diluting with diethyl ether $(20 \mathrm{~mL})$ and pouring into ice-cold water $(20 \mathrm{~mL})$. The ether layer was separated and the aqueous layer was extracted with diethyl ether $(3 \times 30 \mathrm{~mL})$. The combined organic extracts were washed with $10 \% \mathrm{HCl}(2 \times 10 \mathrm{~mL})$, water $(10 \mathrm{~mL})$, brine $(10 \mathrm{~mL})$, dried over anhydrous $\mathrm{Na}_{2} \mathrm{SO}_{4}$, and concentrated under reduced pressure to furnish the crude product $(530 \mathrm{mg})$, which on chromatography over neutral alumina column ( $5 \%$ ethyl acetate/hexane) gave the crystalline trans-diene $5(516 \mathrm{mg}, 52 \%)$. M.p. $140-141^{\circ} \mathrm{C}$; IR (KBr): $\tilde{v}=3052,1731 \mathrm{~cm}^{-1}$; ${ }^{1} \mathrm{H}$ NMR $\left(300 \mathrm{MHz}, \mathrm{CDCl}_{3}\right): \delta=6.09(\mathrm{~m}, 2 \mathrm{H}), 5.91(\mathrm{~m}, 2 \mathrm{H}), 2.56(\mathrm{~s}$, $1 \mathrm{H}), 2.51(\mathrm{~s}, 1 \mathrm{H}), 2.03-1.83(\mathrm{~m}, 2 \mathrm{H}), 1.97(\mathrm{~s}, 6 \mathrm{H}), 1.77-1.43(\mathrm{~m}, 4 \mathrm{H})$; ${ }^{13} \mathrm{C}$ NMR (75 MHz, $\mathrm{CDCl}_{3}$ ): $\delta 169.2$ (2C), 130.8 (2C), 124.1 (2C), 78.8 (2 C), 26.7 (2C), 21.6 (2C), 20.2 (2C); MS (EI, $70 \mathrm{eV}): m / z: 251\left[M^{+}+\mathrm{H}\right]$; elemental analysis calcd (\%) for $\mathrm{C}_{14} \mathrm{H}_{18} \mathrm{O}_{4}$ (250): C 67.18, $\mathrm{H}$ 7.25; found: $\mathrm{C}$, $66.90, \mathrm{H}, 7.52$

Compound 12: Pyridiniumbromide perbromide $(8.22 \mathrm{~g}, 0.025 \mathrm{mmol})$ was added to a solution of the known ${ }^{[10]}$ trans-diol $11(3.6 \mathrm{~g}, 0.023 \mathrm{~mol})$ in dry $\mathrm{CH}_{2} \mathrm{Cl}_{2}(50 \mathrm{~mL})$, and the reaction mixture was stirred at $0{ }^{\circ} \mathrm{C}$ under $\mathrm{N}_{2}$ atmosphere. After consumption of the starting material (monitored by TLC), the reaction mixture was diluted with $\mathrm{CH}_{2} \mathrm{Cl}_{2}(50 \mathrm{~mL})$ and poured into ice-cold water $(100 \mathrm{~mL})$. The organic layer was separated and the aqueous layer was extracted with $\mathrm{CH}_{2} \mathrm{Cl}_{2}(3 \times 30 \mathrm{~mL})$. The combined organic extracts were washed successively with saturated aqueous $\mathrm{Na}_{2} \mathrm{~S}_{2} \mathrm{O}_{5}$ solution $(2 \times 20 \mathrm{~mL})$, water $(20 \mathrm{~mL})$, brine $(20 \mathrm{~mL})$, and dried over anhydrous $\mathrm{Na}_{2} \mathrm{SO}_{4}$. Concentration of the solvent and purification of the resultant solid residue by silica gel chromatography afforded the dibromodiol (4.7 g, 66\%). m.p. $106^{\circ} \mathrm{C}$; IR (thin film): $\tilde{v}=3531,3457 \mathrm{~cm}^{-1}$; ${ }^{1} \mathrm{H}$ NMR (300 MHz, $\left.\mathrm{CDCl}_{3}\right): \delta=4.89(\mathrm{~s}, 2 \mathrm{H}), 2.87(\mathrm{~d}, J=16 \mathrm{~Hz}, 2 \mathrm{H}), 2.47$ $(\mathrm{s}, 2 \mathrm{H}), 2.28(\mathrm{~d}, J=15.6 \mathrm{~Hz}, 2 \mathrm{H}), 1.86-1.80(\mathrm{~m}, 4 \mathrm{H}), 1.62(\mathrm{br} \mathrm{s}, 2 \mathrm{H})$; ${ }^{13} \mathrm{C} \mathrm{NMR}$ (75 MHz, $\mathrm{CDCl}_{3}$ ): $\delta=80.0$ (2 C), 50.1 (2 C), $35.8(2 \mathrm{C}), 33.3$ (2 C), 18.9 (2C); MS (70 eV, EI): $m / z(\%): 314\left[M^{+}\right]$. The dibromodiol was dissolved in acetic anhydride $(10 \mathrm{~mL})$ and $\mathrm{BF}_{3} \cdot \mathrm{Et}_{2} \mathrm{O}(100 \mu \mathrm{L})$ was added at $0{ }^{\circ} \mathrm{C}$ and stirred at the same temperature for $1 \mathrm{~h}$ under $\mathrm{N}_{2}$. On complete consumption of the starting material (monitored by TLC), the reaction mixture was quenched by the careful addition of ice-cold water $(50 \mathrm{~mL})$ and diluted with $\mathrm{CH}_{2} \mathrm{Cl}_{2}(30 \mathrm{~mL})$. The aqueous layer was extracted with $\mathrm{CH}_{2} \mathrm{Cl}_{2}(3 \times 30 \mathrm{~mL})$ and the combined organic extracts were washed carefully with saturated aqueous $\mathrm{NaHCO}_{3}(2 \times 20 \mathrm{~mL})$, brine $(20 \mathrm{~mL})$, and dried over anhydrous $\mathrm{Na}_{2} \mathrm{SO}_{4}$. The residue obtained after concentration at reduced pressure was purified by silica gel chromatography to furnish the dibromodiacetate $\mathbf{1 2}(5.3 \mathrm{~g}, 89 \%)$ as a colorless crystalline solid. M.p. $127.3{ }^{\circ} \mathrm{C}$; IR (thin film): $\tilde{v}=1742 \mathrm{~cm}^{-1} ;{ }^{1} \mathrm{H}$ NMR $\left(300 \mathrm{MHz}, \mathrm{CDCl}_{3}\right): \delta=$ $4.76(\mathrm{~s}, 2 \mathrm{H}), 3.20(\mathrm{brs}, 1 \mathrm{H}), 3.14(\mathrm{brs}, 1 \mathrm{H}), 2.69(\mathrm{~d}, J=3.9 \mathrm{~Hz}, 1 \mathrm{H}), 2.64$ (d, $J=3.9 \mathrm{~Hz}, 1 \mathrm{H}), 2.57-2.53(\mathrm{~m}, 2 \mathrm{H}), 2.01(\mathrm{~s}, 6 \mathrm{H}), 1.8-1.6(\mathrm{~m}, 4 \mathrm{H})$; ${ }^{13} \mathrm{C}$ NMR (75 MHz, $\left.\mathrm{CDCl}_{3}\right): \delta=170.1$ (2C), 86.7 (2C), 48.2 (2C), 30.7 (2C), 27.4 (2C), 23.3 (2C), 18.4; MS (70 eV, EI): $m / z$ (\%): $317\left[\mathrm{M}^{+}-\mathrm{Br}-\right.$ $\mathrm{H}], 319\left[M^{+}-\mathrm{Br}+\mathrm{H}\right]$

$(3 \mathrm{a} R *, 7 \mathrm{a} R *)-2,3,3 \mathrm{a}, 7 \mathrm{a}-\mathrm{Tetrahydro-1H}$-3a,7a-indenediol (6): Dibromodiacetate $12(1 \mathrm{~g}, 2.51 \mathrm{mmol})$ was dissolved in dry DMSO $(5 \mathrm{~mL})$ and DBU $(0.75 \mathrm{~mL}, 5.02 \mathrm{mmol})$ was added dropwise at $5-10^{\circ} \mathrm{C}$ from a syringe under $\mathrm{N}_{2}$ atmosphere, during which time the reaction turned dark brown. The reaction mixture was stirred at room temperature for further $2 \mathrm{~h}$, before diluting with diethyl ether $(30 \mathrm{~mL})$ and pouring into ice-cold water $(10 \mathrm{~mL})$. The organic layer was separated and the aqueous layer was extracted with diethyl ether $(3 \times 50 \mathrm{~mL})$. The combined organic extracts were washed with $10 \% \mathrm{HCl}(2 \times 20 \mathrm{~mL})$, water $(20 \mathrm{~mL})$, brine $(15 \mathrm{~mL})$, dried over anhydrous $\mathrm{Na}_{2} \mathrm{SO}_{4}$, and concentrated under reduced pressure. The resultant solid residue was purified by chromatography over neutral alumina (5\% ethyl acetate/hexane) to furnish crystalline trans-diene 6 (319 mg, 54\%). M.p. $139.5^{\circ} \mathrm{C}$; IR $(\mathrm{KBr}): \tilde{v}=1724 \mathrm{~cm}^{-1} ;{ }^{1} \mathrm{H}$ NMR $\left(300 \mathrm{MHz}, \mathrm{CDCl}_{3}\right): \delta=6.43(\mathrm{~d}, J=10.2 \mathrm{~Hz}, 2 \mathrm{H}), 5.90(\mathrm{~d}, J=10.2 \mathrm{~Hz}$, 2H), $2.41-2.35(\mathrm{~m}, 2 \mathrm{H}), 2.09-1.99(\mathrm{~m}, 2 \mathrm{H}), 1.94-1.84(\mathrm{~m}, 2 \mathrm{H}), 1.94(\mathrm{~s}$, $6 \mathrm{H}) ;{ }^{13} \mathrm{C}$ NMR (75 MHz, $\mathrm{CDCl}_{3}$ ): $\delta=169.6$ (2C), 128.9 (2C), 125.6 (2C), 87.2 (2C), 28.4 (2C), 21.7 (2C), 21.2; MS (70 eV, EI): $\mathrm{m} / z$ (\%): $152\left[\mathrm{M}^{+}-\right.$ $2 \mathrm{Ac}+2]$.

Compounds 13 and 14: $m$ CPBA $(70 \%, 493 \mathrm{mg}, 2 \mathrm{mmol})$ was added at $0{ }^{\circ} \mathrm{C}$ to a solution of $\mathbf{5}(500 \mathrm{mg}, 2 \mathrm{mmol})$ in dry $\mathrm{CH}_{2} \mathrm{Cl}_{2}(10 \mathrm{~mL})$, and the reaction mixture was stirred at room temperature for $5 \mathrm{~h}$ before quenching with $10 \% \mathrm{Na}_{2} \mathrm{SO}_{3}(5 \mathrm{~mL})$. The organic layer was separated and the aqueous layer was extracted with $\mathrm{CH}_{2} \mathrm{Cl}_{2}(3 \times 30 \mathrm{~mL})$. The combined organic layer was washed with saturated aqueous $\mathrm{NaHCO}_{3}(2 \times 10 \mathrm{~mL})$, brine $(10 \mathrm{~mL})$, and dried over anhydrous $\mathrm{Na}_{2} \mathrm{SO}_{4}$. The solvent was removed under reduced pressure to give a solid residue, which by TLC examination was found to be a mixture of two compounds. The residue was subjected to silica gel column chromatography (5\% ethyl acetate/hexane) to furnish epoxides $\mathbf{1 3}$ (295 mg, 66\%) and $\mathbf{1 4}$ (31 mg, $7 \%$ ), along with recovered starting material $5(80 \mathrm{mg})$, in an overall yield of $73 \%$. Compound 13: IR (thin film): $\tilde{v}=$ $1733 \mathrm{~cm}^{-1} ;{ }^{1} \mathrm{H}$ NMR $\left(300 \mathrm{MHz}, \mathrm{CDCl}_{3}\right): \delta=6.13(1 / 2 \mathrm{ABq}, J=9.4,3.7 \mathrm{~Hz}$, $1 \mathrm{H}), 6.08(\mathrm{~d}$ of $1 / 2 \mathrm{ABq}, J=9.4,3.7 \mathrm{~Hz}, 1 \mathrm{H}), 4.23(\mathrm{~d}, J=3.7 \mathrm{~Hz}, 1 \mathrm{H}), 3.33$ (brs, $1 \mathrm{H}), 2.89$ (d, $J=14.2 \mathrm{~Hz}, 1 \mathrm{H}), 2.41(\mathrm{~d}, J=14.2 \mathrm{~Hz}, 1 \mathrm{H}), 2.23-2.09$ $(\mathrm{m}, 1 \mathrm{H}), 2.02(\mathrm{~s}, 3 \mathrm{H}), 2.00(\mathrm{~s}, 3 \mathrm{H}), 1.84-1.75(\mathrm{~m}, 1 \mathrm{H}), 1.52-1.25(\mathrm{~m}, 4 \mathrm{H})$; ${ }^{13} \mathrm{C}$ NMR (75 MHz, $\mathrm{CDCl}_{3}$ ): $\delta=169.3,168.7,134.7,126.2,80.7$ (2C), 55.9, 46.7, 28.5, 25.5, 22.0, 21.7, 20.1, 19.7; MS (EI, $70 \mathrm{eV}): \mathrm{m} / \mathrm{z}(\%): 207\left[\mathrm{M}^{+}{ }_{-}\right.$ OAc]; elemental analysis calcd (\%) for $\mathrm{C}_{14} \mathrm{H}_{18} \mathrm{O}_{5}$ (266.2): C 63.15, H 6.81; found: C $62.85, \mathrm{H} 6.90$.

Compound 14: IR (KBr): $\tilde{v}=1738 \mathrm{~cm}^{-1} ;{ }^{1} \mathrm{H}$ NMR (300 MHz, $\left.\mathrm{CDCl}_{3}\right): \delta=$ $6.15(\mathrm{~d}, J=9.4 \mathrm{~Hz}, 1 \mathrm{H}), 5.95(\mathrm{~d}, J=9.4 \mathrm{~Hz}, 1 \mathrm{H}), 3.41(\mathrm{~d}, J=3.3 \mathrm{~Hz}, 1 \mathrm{H})$, $3.28(\mathrm{~d}, J=3.3 \mathrm{~Hz}, 1 \mathrm{H}), 2.92(\mathrm{~d}, J=13.6 \mathrm{~Hz}, 1 \mathrm{H}), 2.50(\mathrm{~d}, J=13.6 \mathrm{~Hz}, 1 \mathrm{H})$, $2.02(\mathrm{~s}, 6 \mathrm{H}), 1.81-1.25(\mathrm{~m}, 6 \mathrm{H}) ;{ }^{13} \mathrm{C} \mathrm{NMR}\left(75 \mathrm{MHz}, \mathrm{CDCl}_{3}\right): \delta=168.8$, 168.7, 138.2, 124.4, 78.4, 77.7, 54.4, 47.6, 28.3, 27.2, 21.6, 21.2, 20.3, 20.0; MS (70 eV, EI): $m / z(\%): 267\left[M^{+}+\mathrm{H}\right]$; elemental analysis calcd (\%) for $\mathrm{C}_{14} \mathrm{H}_{18} \mathrm{O}_{5}$ (266.2): C 63.15, H 6.81; found: C 62.92, H 6.95.

Compounds 15 and 16: A solution of epoxide $13(250 \mathrm{mg}, 0.94 \mathrm{mmol})$ in THF $(5 \mathrm{~mL})$ was treated with $10 \% \mathrm{AcOH}(2 \mathrm{~mL})$, and the resulting mixture was stirred at $50^{\circ} \mathrm{C}$ for $16 \mathrm{~h}$. Upon completion of the reaction (monitored by TLC), the mixture was cooled and neutralized by the addition of solid $\mathrm{NaHCO}_{3}$, followed by extraction with ethyl acetate $(3 \times$ $30 \mathrm{~mL}$ ). The combined organic extracts were washed with saturated aqueous $\mathrm{NaHCO}_{3}(2 \times 10 \mathrm{~mL})$, brine $(10 \mathrm{~mL})$, dried over anhydrous $\mathrm{Na}_{2} \mathrm{SO}_{4}$, and concentrated under reduced pressure to give a solid. Purification of the residue by column chromatography over silica gel (30\% ethyl acetate/hexane) gave $\mathbf{1 5}(160 \mathrm{mg}, 60 \%)$ and $\mathbf{1 6}(53 \mathrm{mg}, 20 \%)$ as colorless crystalline solids in $80 \%$ overall yield. Compound 15: m.p. $198-$ $199^{\circ} \mathrm{C}$; IR (KBr): $\tilde{v}=3478,1735 \mathrm{~cm}^{-1} ;{ }^{1} \mathrm{H}$ NMR $\left(300 \mathrm{MHz}, \mathrm{CDCl}_{3}\right): \delta=$ 5.98 (ddd, $J=10.2,4.6,2.2 \mathrm{~Hz}, 1 \mathrm{H}), 5.55(\mathrm{dd}, J=10.3,1.3 \mathrm{~Hz}, 1 \mathrm{H}), 5.42$ (d, $J=1.3 \mathrm{~Hz}, 1 \mathrm{H}), 4.68(\mathrm{dd}, J=11.1,4.6 \mathrm{~Hz}, 1 \mathrm{H}), 3.14(\mathrm{~d}, J=11.1 \mathrm{~Hz}, 1 \mathrm{H})$, $2.72(\mathrm{~d}, J=14.7 \mathrm{~Hz}, 1 \mathrm{H}), 2.45(\mathrm{~s}, 1 \mathrm{H}), 2.26-2.05(\mathrm{~m}, 1 \mathrm{H}), 2.16(\mathrm{~s}, 3 \mathrm{H}), 2.04$ $(\mathrm{s}, 3 \mathrm{H}), 1.67-1.56(\mathrm{~m}, 4 \mathrm{H}) ;{ }^{13} \mathrm{C}$ NMR $\left(75 \mathrm{MHz}, \mathrm{CDCl}_{3}\right): \delta=170.1,169.3$, 129.2, 124.6, 83.1 (2 C), 73.6, 72.9, 64.8, 31.7, 24.5, 22.2, 21.0, 20.3; MS (70 eV, EI): $m / z(\%): 285\left[M^{+}+\mathrm{H}\right]$; elemental analysis calcd $(\%)$ for $\mathrm{C}_{14} \mathrm{H}_{20} \mathrm{O}_{6}$ (284): C 59.14, H 7.09; found: C 59.08, H 7.04.

Compound 16: m.p. $162-163^{\circ} \mathrm{C}$; IR $(\mathrm{KBr}): \tilde{v}=3402,1737,1723 \mathrm{~cm}^{-1}$; ${ }^{1} \mathrm{H}$ NMR $\left(300 \mathrm{MHz}, \mathrm{CDCl}_{3}\right): \delta=6.21(\mathrm{~d}, J=10 \mathrm{~Hz}, 1 \mathrm{H}), 5.91(\mathrm{dd}, J=10$, $3.3 \mathrm{~Hz}, 1 \mathrm{H}), 5.54(\mathrm{~d}, J=8.4 \mathrm{~Hz}, 1 \mathrm{H}), 4.29(\mathrm{brs}, 1 \mathrm{H}), 3.04(\mathrm{~d}, J=9.6 \mathrm{~Hz}$, $1 \mathrm{H}), 2.61(\mathrm{~d}, J=11.1 \mathrm{~Hz}, 1 \mathrm{H}), 2.21-2.09(\mathrm{~m}, 2 \mathrm{H}), 2.04(\mathrm{~s}, 3 \mathrm{H}), 2.00(\mathrm{~s}$, $3 \mathrm{H}), 1.87-1.78(\mathrm{~m}, 2 \mathrm{H}), 1.66-1.22(\mathrm{~m}, 4 \mathrm{H}) ;{ }^{13} \mathrm{C} \mathrm{NMR}\left(75 \mathrm{MHz}, \mathrm{CDCl}_{3}\right)$ : $\delta=169.8,167.7,130.1,129.7,80.5,79.9,73.5,70.5,27.7,24.5,22.2,21.8,20.2$, 19.8; MS (70 eV, EI): $m / z(\%): 242\left[M^{+}-\mathrm{Ac}+\mathrm{H}\right]$; elemental analysis calcd (\%) for $\mathrm{C}_{14} \mathrm{H}_{20} \mathrm{O}_{6}$ (284.3): C 59.14, H 7.09; found: C 58.91, H 7.07.

Crystal data for compound 15: Structure was solved by direct methods (SIR92). Refinement was by full-matrix least-squares procedures on $F^{2}$ by using SHELXL-97. Crystal system: rhombohedral-hexagonal, space group: $R 3 c$, cell parameters: $a=27.761(2), b=27.761(2), c=9.956(10) \AA, \gamma=$ $120(6)^{\circ}, V=6645.19 \AA^{3}, Z=18, \rho_{\text {calcd }}=1.279 \mathrm{~g} \mathrm{~cm}^{-3}, F(000)=2736, \mu=$ $0.10 \mathrm{~mm}^{-1}, \lambda=0.71 \AA$. $R 1=0.0491$ for $F_{\mathrm{o}}>2 \sigma\left(F_{\mathrm{o}}\right)$ and 0.0499 for all 2111 data. $w R 2=0.1392, \mathrm{GOF}=1.097$. There are four independent molecules in the asymmetric unit. An ORTEP drawing of compound 15 with $50 \%$ ellipsoidal probability level is shown in Figure 1.

Compound 17: $\mathrm{OsO}_{4}(2 \mathrm{mg}, 1 \mathrm{~mol} \%)$ and $50 \%$ aqueous $N$-methylmorpholine $N$-oxide (NMMO) $(130 \mu \mathrm{L}, 0.56 \mathrm{mmol})$ were added to a solution of diol $15(155 \mathrm{mg}, 0.545 \mathrm{mmol})$ in acetone/water $(4: 1,5 \mathrm{~mL})$ at $0{ }^{\circ} \mathrm{C}$, and the resulting pale yellow reaction mixture was stirred at room temperature for $2 \mathrm{~h}$, before quenching with solid $\mathrm{NaHSO}_{3}$. The resulting mixture was diluted with ethyl acetate $(10 \mathrm{~mL})$, filtered through Celite, and the filtrate was concentrated under reduced pressure. The residue was subjected to column chromatography over silica gel ( $80 \%$ ethyl acetate/hexane) to afford the tetrol $17(152 \mathrm{mg}, 88 \%)$. IR (KBr): $\tilde{v}=3391,1727 \mathrm{~cm}^{-1} ;{ }^{1} \mathrm{H}$ NMR $\left(300 \mathrm{MHz}, \mathrm{D}_{2} \mathrm{O}\right): \delta=5.19(\mathrm{~d}, J=10.2 \mathrm{~Hz}, 1 \mathrm{H}), 5.56(\mathrm{~d}, J=2.5 \mathrm{~Hz}, 1 \mathrm{H})$, $4.10(\mathrm{dd}, J=10.2,4 \mathrm{~Hz}, 1 \mathrm{H}), 4.04(\mathrm{dd}, J=4,2.5 \mathrm{~Hz}, 1 \mathrm{H}), 2.28(\mathrm{~d}, J=$ 


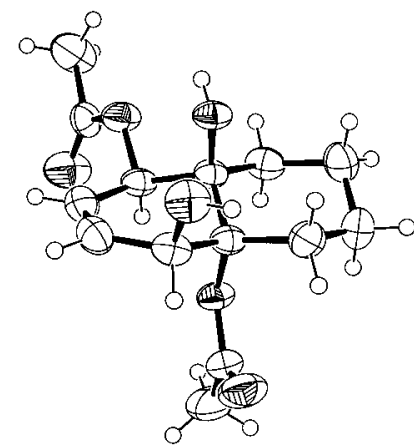

Figure 1. ORTEP drawing for $\mathbf{1 5 .}$

$14.7 \mathrm{~Hz}, 1 \mathrm{H}), 2.06(\mathrm{~s}, 3 \mathrm{H}), 1.95$ (s, 3H), $1.92-1.86(\mathrm{~m}, 1 \mathrm{H}), 1.55-1.21(\mathrm{~m}$, $6 \mathrm{H}) ;{ }^{13} \mathrm{C}$ NMR $\left(75 \mathrm{MHz}, \mathrm{D}_{2} \mathrm{O}\right): \delta=175.5,174.5,83.7,77.5,76.1,74.3,71.2$, 67.4, 30.1, 25.6, 22.9, 21.6, 20.1 (2 C); MS (70 eV, EI): $m / z(\%): 319\left[M^{+}+\mathrm{H}\right]$; elemental analysis calcd (\%) for $\mathrm{C}_{14} \mathrm{H}_{22} \mathrm{O}_{8}(318.3)$ : C 52.82, $\mathrm{H}$ 6.97; found: C 52.63, H 7.07 .

Annulated chiro-inositol 18: Solid $\mathrm{K}_{2} \mathrm{CO}_{3}(78 \mathrm{mg}, 0.566 \mathrm{mmol})$ was added to a solution of tetrol $\mathbf{1 7}(90 \mathrm{mg}, 0.283 \mathrm{mmol})$ in methanol $(3 \mathrm{~mL})$, and the reaction mixture was stirred at room temperature for $2 \mathrm{~h}$, before removal of methanol under reduced pressure. The residue obtained was dissolved in deionized water $(2 \mathrm{~mL})$ and the solution was passed though a Dowex $50 \mathrm{~W} \times 8\left(\mathrm{H}^{+}\right.$form $)$ion exchange resin column and eluted with water. The eluent was concentrated under reduced pressure to give the annulated chiro-inositol 18 (63 mg, $96 \%$ ). M.p. $209-210^{\circ} \mathrm{C}$; IR (KBr): $\tilde{v}=3353 \mathrm{~cm}^{-1}$; ${ }^{1} \mathrm{H}$ NMR $\left(300 \mathrm{MHz}, \mathrm{D}_{2} \mathrm{O}\right): \delta=4.00(\mathrm{dd}, J=3.6,3.3 \mathrm{~Hz}, 1 \mathrm{H}), 3.89(\mathrm{dd}, J=$ $9.9,3.6 \mathrm{~Hz}, 1 \mathrm{H}), 3.61(\mathrm{~d}, J=9.9 \mathrm{~Hz}, 1 \mathrm{H}), 3.55(\mathrm{~d}, J=3.3 \mathrm{~Hz}, 1 \mathrm{H}), 2.05-$ $1.95(\mathrm{~m}, 1 \mathrm{H}), 1.66(\mathrm{~d}, J=13.2 \mathrm{~Hz}, 1 \mathrm{H}), 1.54-1.43(\mathrm{~m}, 5 \mathrm{H}), 1.29(\mathrm{~d}, J=$ $13.2 \mathrm{~Hz}, 1 \mathrm{H}) ;{ }^{13} \mathrm{C}$ NMR $\left(75 \mathrm{MHz}, \mathrm{D}_{2} \mathrm{O}\right): \delta=80.5,78.8,77.4,77.1,75.1,72.3$, 33.0, 32.1, 22.5, 22.1; MS (ES): $m / z$ (\%): $257\left[M^{+}+\mathrm{Na}\right]$.

Crystal data for the compound 18: Structure was solved by direct methods (SIR92). Refinement was by full-matrix least-squares procedures on $F^{2}$ by using SHELXL-97. Crystal system: triclinic, space group: $P \overline{1}$, cell parameters: $a=12.558(3), \quad b=12.915(4), c=13.859(4) \AA, \quad \alpha=87.959(6), \quad \beta=$ $87.627(6), \quad \gamma=70.606(6)^{\circ}, \quad V=2118.20 \AA^{3}, \quad Z=8, \quad \rho_{\text {calcd }}=1.285 \mathrm{~g} \mathrm{~cm}^{-3}$, $F(000)=882, \mu=0.11 \mathrm{~mm}^{-1}, \lambda=0.71 \AA$ $. R 1=0.0512$ for $4439 F_{\mathrm{o}}>4 \sigma\left(F_{\mathrm{o}}\right)$ and 0.0923 for all 7191 data, $w R 2=0.1563, \mathrm{GOF}=0.883$, restrained $\mathrm{GOF}=0.883$ for all data. There are four independent molecules in the asymmetric unit. An ORTEP drawing of compound $\mathbf{1 8}$ with $50 \%$ ellipsoidal probability level is shown in Figure 2.

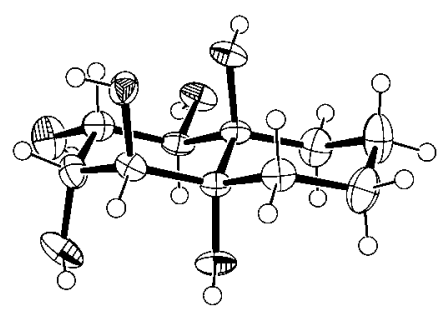

Figure 2. ORTEP drawing for 18.

Compound 19: The tetrol $\mathbf{1 9}$ ( $35 \mathrm{mg}, 95 \%$ ) was obtained from diacetate $\mathbf{1 6}$ (53 $\mathrm{mg}, 0.187 \mathrm{mmol}$ ) by following a procedure similar to that described for the conversion of $\mathbf{1 7}$ to $\mathbf{1 8}$ but using $\mathrm{K}_{2} \mathrm{CO}_{3}(51 \mathrm{mg}, 0.374 \mathrm{mmol})$ in methanol $(2 \mathrm{~mL})$ and purification over a silica gel $(50 \%$ ethyl acetate/ hexane). M.p. $202-203{ }^{\circ} \mathrm{C}$; IR (thin film): $\tilde{v}=3273 \mathrm{~cm}^{-1} ;{ }^{1} \mathrm{H}$ NMR $\left(300 \mathrm{MHz}, \mathrm{D}_{2} \mathrm{O}\right): \delta=5.71(\mathrm{~d} 1 / 2 \mathrm{ABq}, J=10,3.8 \mathrm{~Hz}, 1 \mathrm{H}), 5.61(1 / 2 \mathrm{ABq}$, $J=10,3.8 \mathrm{~Hz}, 1 \mathrm{H}), 3.97(\mathrm{~d}, J=3.8 \mathrm{~Hz}, 1 \mathrm{H}), 3.53(\mathrm{~s}, 1 \mathrm{H}), 2.09-2.00(\mathrm{~m}$, $1 \mathrm{H}), 1.65-1.29(\mathrm{~m}, 7 \mathrm{H}) ;{ }^{13} \mathrm{C} \mathrm{NMR}\left(75 \mathrm{MHz}, \mathrm{D}_{2} \mathrm{O}\right): \delta=134.6,127.9,77.7$, 71.4, 70.9, 70.6, 32.7, 30.3, 20.4, 19.9; MS (70 eV, EI): $m / z(\%): 164\left[M^{+}-\right.$ $2 \mathrm{H}_{2} \mathrm{O}$ ]; elemental analysis calcd (\%) for $\mathrm{C}_{10} \mathrm{H}_{16} \mathrm{O}_{4}$ (200.2): C 59.98, H 8.05; found: C 59.79, H 7.89.
Annulated myo-inositol 4: The annulated inositol 4 (18 mg, $80 \%)$ was obtained from the olefin $19(20 \mathrm{mg}, 0.1 \mathrm{mmol})$ by dihydroxylation following the procedure described above for the conversion of $\mathbf{1 5}$ to $\mathbf{1 7}$ but using $\mathrm{OsO}_{4}(1 \mathrm{mg}, 5 \mathrm{~mol} \%)$ and NMMO $(30 \mu \mathrm{L}, 0.128 \mathrm{mmol})$ in acetone/water $(4: 1,1 \mathrm{~mL})$ and purification by silica gel column chromatography $(10 \%$ methanol/ethyl acetate). M.p. $196-197^{\circ} \mathrm{C}$; IR (KBr): $\tilde{v}=3308 \mathrm{~cm}^{-1}$; ${ }^{1} \mathrm{H}$ NMR $\left(300 \mathrm{MHz}, \mathrm{D}_{2} \mathrm{O}\right): \delta=3.98($ brs, $1 \mathrm{H}), 3.94(\mathrm{~m}, 1 \mathrm{H}), 3.74(\mathrm{~m}$, $1 \mathrm{H}), 3.42(\mathrm{brs}, 1 \mathrm{H}), 2.08-2.00(\mathrm{~m}, 1 \mathrm{H}), 1.65(\mathrm{brd}, J=9.3 \mathrm{~Hz}, 1 \mathrm{H}), 1.51-$ $1.28(\mathrm{~m}, 5 \mathrm{H}), 1.24(\mathrm{~d}, J=14 \mathrm{~Hz}, 1 \mathrm{H}) ;{ }^{13} \mathrm{C} \mathrm{NMR}\left(75 \mathrm{MHz}, \mathrm{D}_{2} \mathrm{O}\right): \delta 77.3$, 76.2, 75.6, 74.8, 74.1, 68.4, 30.5, 30.1, 20.1, 19.6; MS (ES): $m / z(\%): 257$ $\left[M^{+}+\mathrm{Na}\right]$.

Crystal data for the compound 4: Structure was solved by direct methods (SIR92). Refinement was by full-matrix least-squares procedures on $F^{2}$ by using SHELXL-97. Crystal system: triclinic, space group: $P \overline{1}$, cell parameters: $a=6.113(1), \quad b=7.612(1), \quad c=11.667(2) \AA, \quad \alpha=102.326(4), \quad \beta=$ 97.912(4), $\quad \gamma=91.817(4)^{\circ}, \quad V=524.39 \AA^{3}, \quad Z=2, \quad \rho_{\text {calcd }}=1.484 \mathrm{~g} \mathrm{~cm}^{-3}$, $F(000)=252.0, \mu=0.12 \mathrm{~mm}^{-1}, \lambda=0.71 \AA . \quad R 1=0.0498$ for $892 F_{\mathrm{o}}>4 \sigma\left(F_{\mathrm{o}}\right)$ and 0.0788 for all 1273 data $w R 2=0.0963, \mathrm{GOF}=1.100$, restrained GOF $=$ 1.100 for all data. An ORTEP drawing of compound $\mathbf{4}$ with $50 \%$ ellipsoidal probability level is shown in Figure 3.

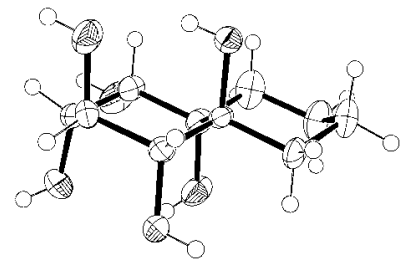

Figure 3. ORTEP drawing for 4.

Compounds 20 and 21: A solution of the diene $5(100 \mathrm{mg}, 0.4 \mathrm{mmol})$ in acetone/water $(4: 1,5 \mathrm{~mL})$ was treated with $\mathrm{OsO}_{4}(1 \mathrm{mg}, 1 \mathrm{~mol} \%)$ and NMMO $(190 \mu \mathrm{L}, 0.8 \mathrm{mmol})$, and the resulting solution was stirred at $10^{\circ} \mathrm{C}$ for $2 \mathrm{~d}$. The reaction was quenched by the addition of solid $\mathrm{NaHSO}_{3}$. After dilution with ethyl acetate $(20 \mathrm{~mL})$, the reaction mixture was filtered through a Celite pad, and the filtrate was concentrated under reduced pressure to give a solid residue, which was indicated to be a mixture of two compounds by TLC. The isomers $\mathbf{2 0}$ and $\mathbf{2 1}$ were separated by chromatography over a silica gel (40\% ethyl acetate/hexane) to furnish $20(53 \mathrm{mg}$, $47 \%$ ) and 21 (26 mg, $23 \%)$.

Compound 20: m.p. $210-211^{\circ} \mathrm{C}$; IR (KBr): $\tilde{v}=3419,1731 \mathrm{~cm}^{-1} ;{ }^{1} \mathrm{H}$ NMR $\left(300 \mathrm{MHz}, \mathrm{D}_{2} \mathrm{O}\right): \delta=4.50(\mathrm{~s}, 2 \mathrm{H}), 3.78(\mathrm{~s}, 2 \mathrm{H}), 2.38(\mathrm{~s}, 1 \mathrm{H}), 2.34(\mathrm{~s}, 1 \mathrm{H})$, $2.01-1.97(\mathrm{~m}, 2 \mathrm{H}), 1.96(\mathrm{~s}, 6 \mathrm{H}), 1.5-1.4(\mathrm{~m}, 2 \mathrm{H}), 1.13-1.06(\mathrm{~m}, 2 \mathrm{H})$; ${ }^{13} \mathrm{C}$ NMR (75 MHz, D $\left.2 \mathrm{O}\right): \delta=172.5$ (2C), 86.0 (2C), 71.4 (2C), 67.4 (2C), 24.8 (2C), 22.2 (2C), 19.5 (2C); MS (70 eV, EI): $m / z(\%): 223\left[\mathrm{M}^{+}{ }_{-}\right.$ $\left.2 \mathrm{H}_{2} \mathrm{O}-\mathrm{OAc}\right]$; elemental analysis calcd (\%) for $\mathrm{C}_{14} \mathrm{H}_{22} \mathrm{O}_{8}$ (318.3): C 52.82, H 6.97; found: C 52.96, H 7.27.

Compound 21: m.p. $215-216^{\circ} \mathrm{C}$; IR (thin film): $3419 \mathrm{~cm}^{-1} ;{ }^{1} \mathrm{H}$ NMR $\left(300 \mathrm{MHz}, \mathrm{D}_{2} \mathrm{O}\right): \delta=5.12(\mathrm{dd}, J=10.6,3 \mathrm{~Hz}, 1 \mathrm{H}), 4.89(\mathrm{brs}, 1 \mathrm{H}), 4.28-$ $4.20(\mathrm{~m}, 2 \mathrm{H}), 2.79$ (br s, $1 \mathrm{H}), 2.60-2.55(\mathrm{~m}, 2 \mathrm{H}), 2.16(\mathrm{~s}, 3 \mathrm{H}), 2.14(\mathrm{~s}, 3 \mathrm{H})$, $1.85-1.30(\mathrm{~m}, 5 \mathrm{H}) ;{ }^{13} \mathrm{C}$ NMR $\left(75 \mathrm{MHz}, \mathrm{D}_{2} \mathrm{O}\right): \delta=170.9,168.4,85.0,78.4$, 74.1, 71.7, 71.6, 66.8, 30.3, 24.6, 22.2, 21.1, 19.9, 19.1; MS (70 eV, EI): $\mathrm{m} / \mathrm{z}$ (\%): $283\left[M^{+}-2 \mathrm{H}_{2} \mathrm{O}+\mathrm{H}\right]$.

Annulated chiro-inositol 22: By following a procedure similar to that used for the conversion of $\mathbf{1 7}$ to $\mathbf{1 8}$, tetrols $\mathbf{2 0}(45 \mathrm{mg}, 0.158 \mathrm{mmol})$ and $\mathbf{2 1}(25 \mathrm{mg}$, $0.088 \mathrm{mmol}$ ) were separately hydrolyzed using $\mathrm{K}_{2} \mathrm{CO}_{3}(43 \mathrm{mg}, 0.316 \mathrm{mmol}$ for 20, $24 \mathrm{mg}, 0.176 \mathrm{mmol}$ for 21) in methanol to afford the annulated inositol 22 (34 mg, $18 \mathrm{mg}$, respectively, $90 \%$ ). IR (thin film): $3364 \mathrm{~cm}^{-1}$; ${ }^{1} \mathrm{H}$ NMR $\left(300 \mathrm{MHz}, \mathrm{D}_{2} \mathrm{O}\right): \delta=3.95(\mathrm{~s}, 2 \mathrm{H}), 3.51(\mathrm{~s}, 2 \mathrm{H}), 2.00-1.91(\mathrm{~m}$, $2 \mathrm{H}), 1.52-1.38(\mathrm{~m}, 4 \mathrm{H}), 1.18(\mathrm{~s}, 1 \mathrm{H}), 1.14(\mathrm{~s}, 1 \mathrm{H}) ;{ }^{13} \mathrm{C} \mathrm{NMR}(75 \mathrm{MHz}$, $\mathrm{D}_{2} \mathrm{O}$ ): $\delta=78.8$ (2C), 74.5 (2C), 69.2 (2C), 30.6 (2C), 19.8 (2C); MS (EI, $70 \mathrm{eV}): m / z(\%): 217\left[M^{+}-\mathrm{H}_{2} \mathrm{O}+\mathrm{H}\right]$.

Compound 23 and 24: Mono-epoxidation of 6 was carried out by following a procedure similar to that described for $\mathbf{5}$ by treating the diene $(1.2 \mathrm{~g}$, $5.08 \mathrm{mmol})$ with $m \mathrm{CPBA}(70 \%, 1.25 \mathrm{~g}, 5.08 \mathrm{mmol})$ in dry $\mathrm{CH}_{2} \mathrm{Cl}_{2}(10 \mathrm{~mL})$ at $0{ }^{\circ} \mathrm{C}$, followed by chromatography over silica gel (elution with $10 \%$ ethyl acetate/hexane) to furnish mono-epoxides $\mathbf{2 3}(537 \mathrm{mg}, 42 \%)$ and $\mathbf{2 4}$ (230 mg, 18\% ). 
Compound 23: m.p. $167^{\circ} \mathrm{C}$; IR (KBr): $\tilde{v}=1730 \mathrm{~cm}^{-1} ;{ }^{1} \mathrm{H}$ NMR $(300 \mathrm{MHz}$, $\left.\mathrm{CDCl}_{3}\right): \delta=6.44(\mathrm{~d}, J=9.3 \mathrm{~Hz}, 1 \mathrm{H}), 6.06(\mathrm{dd}, J=9.8 \mathrm{~Hz}, 3.9 \mathrm{~Hz}, 1 \mathrm{H}), 4.31$ $(\mathrm{d}, J=4.2 \mathrm{~Hz}, 1 \mathrm{H}), 3.31-3.29(\mathrm{~m}, 1 \mathrm{H}), 2.68-2.61(\mathrm{~m}, 1 \mathrm{H}), 2.34-2.24(\mathrm{~m}$, $2 \mathrm{H}), 1.97-1.79(\mathrm{~m}, 9 \mathrm{H}) ;{ }^{13} \mathrm{C}$ NMR $\left(75 \mathrm{MHz}, \mathrm{CDCl}_{3}\right): \delta=169.5,169.1$, 133.1, 127.4, 89.1, 86.1, 51.8, 47.5, 30.1, 27.4, 22.0, 21.9, 20.7; MS (70 eV, EI): $\mathrm{m} / \mathrm{z}(\%): 209\left[\mathrm{M}^{+}-\mathrm{Ac}\right]$; elemental analysis calcd (\%) for $\mathrm{C}_{13} \mathrm{H}_{16} \mathrm{O}_{5}(252.2)$ : C 61.09, H 6.39; found: C 61.47, H 6.36 .

Copmound 24: m.p. $170{ }^{\circ} \mathrm{C}$; IR (thin film): $\tilde{v}=1731 \mathrm{~cm}^{-1} ;{ }^{1} \mathrm{H}$ NMR $\left(300 \mathrm{MHz}, \mathrm{CDCl}_{3}\right): \delta=6.47(\mathrm{~d}, J=9.9 \mathrm{~Hz}, 1 \mathrm{H}), 5.95(\mathrm{~d}, J=9.9 \mathrm{~Hz}, 1 \mathrm{H})$, $3.68(\mathrm{~d}, J=3.6 \mathrm{~Hz}, 1 \mathrm{H}), 3.29(\mathrm{~s}, 1 \mathrm{H}), 2.75-2.66(\mathrm{~m}, 1 \mathrm{H}), 2.43-2.35(\mathrm{~m}$, $1 \mathrm{H}), 2.17-1.75(\mathrm{~m}, 4 \mathrm{H}), 1.97(\mathrm{~s}, 6 \mathrm{H}) ;{ }^{13} \mathrm{C}$ NMR $\left(75 \mathrm{MHz}, \mathrm{CDCl}_{3}\right): \delta=$ 169.1, 168.9, 134.8, 125.3, 86.4, 85.5, 52.7, 48.0, 30.5, 29.3, 21.7, 21.2, 19.2; MS(70 eV, EI): $m / z(\%): 167\left[M^{+}-2 \mathrm{Ac}+\mathrm{H}\right]$.

Compounds 25 and 26: Epoxide 23 (500 $\mathrm{mg}, 1.98 \mathrm{mmol}$ ) was transformed into diols $\mathbf{2 5}(246 \mathrm{mg}, 46 \%)$ and $\mathbf{2 6}(75 \mathrm{mg}, 14 \%)$ by following a procedure similar to that used for the conversion of the epoxide $\mathbf{1 3}$ into diols $\mathbf{1 5}$ and $\mathbf{1 6}$ but using $10 \% \mathrm{AcOH}(3 \mathrm{~mL})$ and separation of the isomers by chromatography over silica gel ( $40 \%$ ethyl acetate/hexane).

Compound 25: m.p. $99.6^{\circ} \mathrm{C}$; IR (KBr): $\tilde{v}=3437,1744,1731 \mathrm{~cm}^{-1} ;{ }^{1} \mathrm{H}$ NMR $\left(300 \mathrm{MHz}, \mathrm{CDCl}_{3}\right): \delta=6.02-5.96(\mathrm{~m}, 1 \mathrm{H}), 5.62-5.57(\mathrm{~m}, 2 \mathrm{H}), 4.80(\mathrm{dd}$, $J=10.8,4.8 \mathrm{~Hz}, 1 \mathrm{H}), 2.64-2.53(\mathrm{~m}, 3 \mathrm{H}), 2.15(\mathrm{~s}, 3 \mathrm{H}), 1.96(\mathrm{~s}, 3 \mathrm{H}), 1.96-$ $1.90(\mathrm{~m}, 1 \mathrm{H}), 1.80-1.70(\mathrm{~m}, 2 \mathrm{H}) ;{ }^{13} \mathrm{C}$ NMR $\left(75 \mathrm{MHz}, \mathrm{CDCl}_{3}\right): \delta=170.0$, 169.4, 130.6, 125.5, 90.5, 81.4, 73.6, 64.5, 34.7, 26.6, 22.2, 21.1, 18.8; MS (70 eV, EI): $m / z(\%): 210\left[M^{+}-\mathrm{Ac}-\mathrm{H}_{2} \mathrm{O}+\mathrm{H}\right]$; elemental analysis calcd (\%) for $\mathrm{C}_{13} \mathrm{H}_{18} \mathrm{O}_{6}$ (270.2): C 57.77, H 6.71; found: C 57.61, H 6.70.

Compound 26: m.p. $150.3{ }^{\circ} \mathrm{C}$; IR (KBr): $\tilde{v}=3467,1730,1711 \mathrm{~cm}^{-1} ;{ }^{1} \mathrm{H}$ NMR $\left(300 \mathrm{MHz}, \mathrm{CDCl}_{3}\right): \delta=6.42(\mathrm{~d}, J=9.6 \mathrm{~Hz}, 1 \mathrm{H}), 5.88(\mathrm{dd}, J=10.1,3 \mathrm{~Hz}$, $1 \mathrm{H}), 4.55(\mathrm{brs}, 1 \mathrm{H}), 4.40(\mathrm{brs}, 1 \mathrm{H}), 2.59-2.28(\mathrm{~m}, 3 \mathrm{H}), 2.11-1.95(\mathrm{~m}, 1 \mathrm{H})$, $1.99(\mathrm{~s}, 3 \mathrm{H}), 1.95(\mathrm{~s}, 3 \mathrm{H}), 2.11-1.95(\mathrm{~m}, 1 \mathrm{H}), 1.89-1.85(\mathrm{~m}, 1 \mathrm{H}) ;{ }^{13} \mathrm{C} \mathrm{NMR}$ (75 MHz, $\mathrm{CDCl}_{3}$ ): $\delta=169.9,168.1,131.9,127.8,89.6,88.6,73.2,72.9,30.02$, 25.9, 22.3, 21.7, 19.8; MS (70 eV, EI): $m / z(\%): 228\left[M^{+}-\mathrm{Ac}+\mathrm{H}\right]$.

Crystal data for the compound 25: Structure was solved by direct methods (SIR92). Refinement was by full-matrix least-squares procedures on $F^{2}$ by using SHELXL-97. Crystal system: monoclinic, space group: $P 12_{1} / m 1$, cell parameters: $a=10.4616(4), b=10.476(4), c=12.454(5) \AA, \beta=101.117(8)^{\circ}$, $V=1339.40 \AA^{3}, Z=4, \rho_{\text {calcd }}=1.34 \mathrm{~g} \mathrm{~cm}^{-3}, F(000)=576.0, \mu=0.11 \mathrm{~mm}^{-1}$, $\lambda=0.71073 \AA$. Total number of 1.s. parameters $=433$. $R 1=0.1012$ for $1953 F_{\mathrm{o}}>4 \sigma\left(F_{\mathrm{o}}\right)$ and 0.2277 for all 3949 data. $w R 2=0.2523$, GOF $=1.252$, restrained $\mathrm{GOF}=1.252$ for all data. An ORTEP drawing of compound 25 with $50 \%$ ellipsoidal probability is shown in Figure 4. There are two independent molecules in the asymmetric unit.

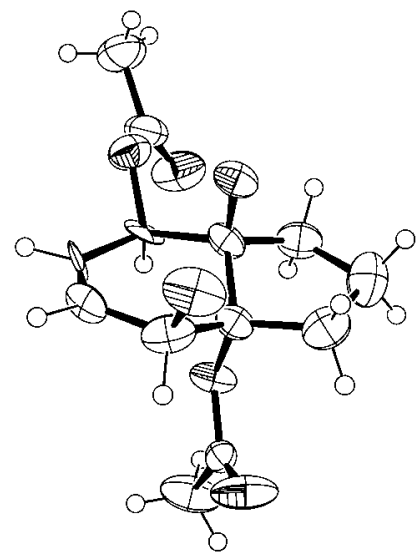

Figure 4. ORTEP drawing for $\mathbf{2 5}$.

Crystal data for the compound 26: Structure was solved by direct methods (SIR92). Refinement was by full-matrix least-squares procedures on $F^{2}$ by using SHELXL-97. Crystal system: monoclinic, space group: $P 2_{1} / c$, cell parameters: $a=7.949(3), b=22.668(11), c=7.970(3) \AA ⿻ 1$. $V=1325.81 \AA^{3}, Z=4, \rho_{\text {calcd }}=1.354 \mathrm{~g} \mathrm{~cm}^{-3}, F(000)=576.0, \mu=0.11 \mathrm{~mm}^{-1}$, $\lambda=0.71073 \AA$. Total number of 1.s. parameters $=244 . R 1=0.0832$ for $2250 F_{\mathrm{o}}>4 \sigma\left(F_{\mathrm{o}}\right)$ and 0.1002 for all 2680 data. $w R 2=0.1882, \mathrm{GOF}=1.227$,

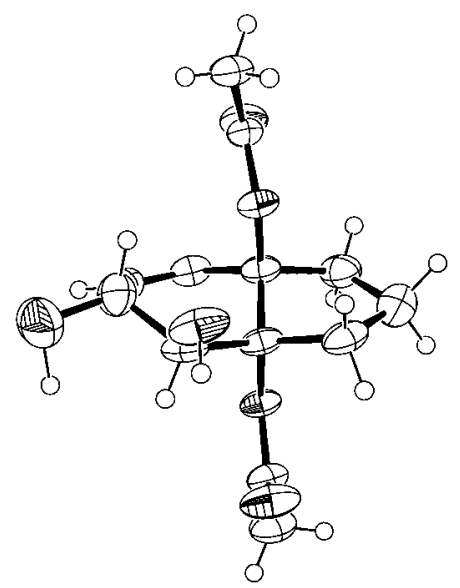

Figure 5. ORTEP drawing for 26.

restrained GOF $=1.227$ for all data. An ORTEP drawing of compound $\mathbf{2 6}$ with $50 \%$ ellipsoidal probability is shown in Figure 5.

Compound 27: Diol 25 ( $230 \mathrm{mg}, 0.85 \mathrm{mmol}$ ) was hydrolyzed with $\mathrm{K}_{2} \mathrm{CO}_{3}$ $(234 \mathrm{mg}, 1.7 \mathrm{mmol})$ in methanol $(3 \mathrm{~mL})$ at room temperature for $6 \mathrm{~h}$. Removal of the solvent under reduced pressure gave a solid residue which was purified by chromatography over silica gel ( $70 \%$ ethyl acetate/hexane) to furnish the tetrol $27(128 \mathrm{mg}, 81 \%)$. IR (KBr): $\tilde{v}=3399 \mathrm{~cm}^{-1} ;{ }^{1} \mathrm{H}$ NMR $\left(300 \mathrm{MHz}, \mathrm{D}_{2} \mathrm{O}\right): \delta=5.86-5.81(\mathrm{~m}, 1 \mathrm{H}), 5.65(\mathrm{~d}, J=9.9 \mathrm{~Hz}, 1 \mathrm{H}), 4.23(\mathrm{~s}$, $1 \mathrm{H}), 3.92(\mathrm{~s}, 1 \mathrm{H}), 2.29-2.24(\mathrm{~m}, 1 \mathrm{H}), 1.78($ br s, $4 \mathrm{H}), 1.58-1.52(\mathrm{~m}, 1 \mathrm{H})$; ${ }^{13} \mathrm{C}$ NMR $\left(75 \mathrm{MHz}, \mathrm{D}_{2} \mathrm{O}\right): \delta=131.9,128.8,82.5,81.9,71.4,69.7,33.9,31.6$, 18.9; MS (70 eV, EI): $m / z$ (\%): $150\left[M^{+}-2 \mathrm{H}_{2} \mathrm{O}\right]$; elemental analysis calcd (\%) for $\mathrm{C}_{9} \mathrm{H}_{14} \mathrm{O}_{4}$ (186.2): C 58.05, H 7.58; found: C 58.14, H 7.71.

Annulated chiro-inositol 28: The tetrol 27 (128 mg, $0.688 \mathrm{mmol})$ was dihydroxylated by following a procedure similar to that described for 17 but using $\mathrm{OsO}_{4}(2 \mathrm{mg}, 1 \mathrm{~mol} \%)$ and NMMO $(175 \mu \mathrm{L}, 0.75 \mathrm{mmol})$ in acetone/ water $(4: 1,3 \mathrm{~mL})$, followed by chromatography over silica gel $(10 \%$ methanol/ethyl acetate) to afford the annulated chiro-inositol $\mathbf{2 8}(127 \mathrm{mg}$, $84 \%)$. IR (KBr): $\tilde{v}=3364 \mathrm{~cm}^{-1}$; ${ }^{1} \mathrm{H}$ NMR $\left(300 \mathrm{MHz}, \mathrm{D}_{2} \mathrm{O}\right): \delta=4.02(\mathrm{~s}$, $1 \mathrm{H}), 3.96-3.81(\mathrm{~m}, 3 \mathrm{H}), 2.22-2.11(\mathrm{~m}, 1 \mathrm{H}), 1.89-1.77(\mathrm{~m}, 3 \mathrm{H}), 1.64-1.61$ $(\mathrm{m}, 1 \mathrm{H}), 1.51-1.45(\mathrm{~m}, 1 \mathrm{H}) ;{ }^{13} \mathrm{C}$ NMR $\left(75 \mathrm{MHz}, \mathrm{D}_{2} \mathrm{O}\right): \delta=86.9,83.7,75.8$, 72.6, 72.4, 70.7, 33.2, 31.3, 19.7; MS (70 eV, EI): $m / z(\%): 184\left[M^{+}-2 \mathrm{H}_{2} \mathrm{O}\right]$; elemental analysis calcd (\%) for $\mathrm{C}_{9} \mathrm{H}_{16} \mathrm{O}_{6}$ (220.2): C, 49.09, $\mathrm{H}$ 7.32; found: $\mathrm{C}$ 48.69, H 7.29 .

Compound 29: Diol diacetate 26 ( $75 \mathrm{mg}, 0.278 \mathrm{mmol}$ ) was hydrolyzed with $\mathrm{K}_{2} \mathrm{CO}_{3}(76 \mathrm{mg}, 0.556 \mathrm{mmol})$ in methanol $(2 \mathrm{~mL})$ by following a procedure similar to that used for the conversion of $\mathbf{1 6}$ to 19. Purification of the resultant residue over silica gel $(70 \%$ ethyl acetate/hexane) furnished 29 (47 mg, $92 \%$ ). M.p. $124.6{ }^{\circ} \mathrm{C}$; IR (thin film): $\tilde{v}=3338 \mathrm{~cm}^{-1} ;{ }^{1} \mathrm{H}$ NMR $\left(300 \mathrm{MHz}, \mathrm{D}_{2} \mathrm{O}\right): \delta=5.99(\mathrm{~d}, J=10.2,1 \mathrm{H}), 5.76(\mathrm{~d}, J=7.2 \mathrm{~Hz}, 1 \mathrm{H}), 4.15(\mathrm{~s}$, $1 \mathrm{H}), 3.83(\mathrm{~s}, 1 \mathrm{H}), 2.17-2.06(\mathrm{~m}, 1 \mathrm{H}), 1.81-1.77(\mathrm{~m}, 3 \mathrm{H}), 1.63-1.48(\mathrm{~m}$, $2 \mathrm{H}) ;{ }^{13} \mathrm{C}$ NMR (75 MHz, $\mathrm{D}_{2} \mathrm{O}$ ): $\delta=131.0,130.6,80.3,79.9,75.8,72.6,33.4$, 29.94, 19.8; MS (70 eV, EI): $m / z(\%): 167\left[M^{+}-\mathrm{H}_{2} \mathrm{O}-\mathrm{H}\right]$.

Annulated myo-inositol 30: Tetrol 29 (45 mg, $0.242 \mathrm{mmol})$ was dihydroxylated with $\mathrm{OsO}_{4}(1 \mathrm{mg}, 2 \mathrm{~mol} \%)$ and NMMO $(70 \mu \mathrm{L}, 0.29 \mathrm{mmol})$ in acetone/water $(4: 1,2 \mathrm{~mL})$ following a procedure described above for the conversion of $\mathbf{1 9}$ to $\mathbf{4}$. Chromatography of the resultant residue over silica gel $(10 \%$ methanol/ethyl acetate) afforded annulated myo-inositol 30 (45 mg, $86 \%$ ). IR (KBr): $\tilde{v}=3386 \mathrm{~cm}^{-1} ;{ }^{1} \mathrm{H}$ NMR (300 MHz, $\left.\mathrm{D}_{2} \mathrm{O}\right): \delta=$ $4.06(\mathrm{~s}, 1 \mathrm{H}), 3.96(\mathrm{~s}, 2 \mathrm{H}), 3.79(\mathrm{~s}, 1 \mathrm{H}), 2.20-2.13(\mathrm{~m}, 1 \mathrm{H}), 1.80-1.61(\mathrm{~m}$, $4 \mathrm{H}), 1.47-1.42(\mathrm{~m}, 1 \mathrm{H}) ;{ }^{13} \mathrm{C}$ NMR $\left(75 \mathrm{MHz}, \mathrm{D}_{2} \mathrm{O}\right): \delta=85.8,84.7,76.5$, 74.6, 73.7, 68.50, 33.6, 31.6, 18.7; MS (70 eV, EI): $m / z(\%): 166\left[M^{+}-\right.$ $\left.3 \mathrm{H}_{2} \mathrm{O}\right]$.

Compound 31: Epoxide 24 ( $225 \mathrm{mg}, 0.893 \mathrm{mmol}$ ) was converted to the diol 31 (178 $\mathrm{mg}, 74 \%)$ by following a procedure similar to that used for the major epoxide 23 but with $10 \% \mathrm{AcOH}(3 \mathrm{~mL})$ and purification over silica gel $\left(40 \%\right.$ ethyl acetate/hexane). M.p. $124.5^{\circ} \mathrm{C}$; IR $(\mathrm{KBr}): \tilde{v}=3452$, $1716 \mathrm{~cm}^{-1} ;{ }^{1} \mathrm{H}$ NMR $\left(300 \mathrm{MHz}, \mathrm{CDCl}_{3}\right): \delta=5.69(\mathrm{~s}, 2 \mathrm{H}), 5.67(\mathrm{~s}, 2 \mathrm{H})$, $2.14(\mathrm{~s}, 6 \mathrm{H}), 2.21-2.05(\mathrm{~m}, 2 \mathrm{H}), 1.96-1.83(\mathrm{~m}, 2 \mathrm{H}), 1.75-1.67(\mathrm{~m}, 2 \mathrm{H})$; ${ }^{13} \mathrm{C}$ NMR (75 MHz, $\mathrm{CDCl}_{3}$ ): $\delta=170.0$ (2C), 128.0 (2C), 83.3 (2C), 73.8 
(2C), 34.7 (2C), 21.1 (2C), 19.4; MS (70 eV, EI): $\mathrm{m} / \mathrm{z}(\%): 210\left[\mathrm{M}^{+}-\right.$ $\left.\mathrm{AcO}-\mathrm{H}_{2} \mathrm{O}+\mathrm{H}\right]$.

Compound 32: The diol diacetate 31 (175 mg, $0.648 \mathrm{mmol}$ ) was hydrolyzed with $\mathrm{K}_{2} \mathrm{CO}_{3}(178 \mathrm{mg}, 1.296 \mathrm{mmol})$ in methanol $(3 \mathrm{~mL})$ by following a procedure similar to that used for the conversion of $\mathbf{1 6}$ to $\mathbf{1 9}$ to afford the tetrol 32 (114 mg, $95 \%$ ). M.p. $114{ }^{\circ} \mathrm{C}$; IR (KBr): $\tilde{v}=3400 \mathrm{~cm}^{-1} ;{ }^{1} \mathrm{H}$ NMR $\left(300 \mathrm{MHz}, \mathrm{D}_{2} \mathrm{O}\right): \delta=5.49(\mathrm{~s}, 2 \mathrm{H}), 4.21(\mathrm{~s}, 2 \mathrm{H}), 1.91-1.71(\mathrm{~m}, 6 \mathrm{H})$; ${ }^{13} \mathrm{C}$ NMR (75 MHz, $\mathrm{D}_{2} \mathrm{O}$ ): $\delta=131.0$ (2C), $85.2(2 \mathrm{C}), 71.2$ (2C), 33.9 (2C), 19.5; MS (70 eV, EI): $m / z$ (\%): $150\left[M^{+}-2 \mathrm{H}_{2} \mathrm{O}\right]$.

Annulated allo-inositol 33: Tetrol $32(100 \mathrm{mg}, 0.537 \mathrm{mmol})$ was dihydroxylated by using $\mathrm{OsO}_{4}(1 \mathrm{mg}, 1 \mathrm{~mol} \%)$ and $\mathrm{NMMO}(150 \mu \mathrm{L}, 0.644 \mathrm{mmol})$ by following a procedure similar to that used for the conversion of $\mathbf{1 9}$ to $\mathbf{4}$. Purification by chromatography over silica gel $(10 \%$ methanol/ethyl acetate) led to 33. M.p. $237^{\circ} \mathrm{C}$; IR (KBr): $\tilde{\nu}=3382 \mathrm{~cm}^{-1} ;{ }^{1} \mathrm{H}$ NMR $\left(300 \mathrm{MHz}, \mathrm{D}_{2} \mathrm{O}\right): \delta=3.96(\mathrm{~s}, 1 \mathrm{H}), 3.78-3.68(\mathrm{~m}, 3 \mathrm{H}), 1.87-1.59(\mathrm{~m}, 6 \mathrm{H})$; ${ }^{13} \mathrm{C}$ NMR (75 MHz, $\left.\mathrm{D}_{2} \mathrm{O}\right): \delta=87.7,85.1,75.7,72.6,72.2,68.8,33.2,32.9,19.7$.

Crystal data for compound 33: Structure was solved by direct methods (SIR92). Refinement was by full-matrix least-squares procedures on $F^{2}$ by using SHELXL-97. Crystal system: monoclinic, space group: $P 12_{1} 1$, cell parameters: $\quad a=7.221(17), \quad b=11.507(27), \quad c=7.291(17) \AA, \quad \beta=$ $103.692(31)^{\circ}, V=588.63 \AA^{3}, Z=2, \quad \rho_{\text {calcd }}=1.242 \mathrm{~g} \mathrm{~cm}^{-3}, \quad F(000)=236.0$, $\mu=0.10 \mathrm{~mm}^{-1}, \lambda=0.71073 \AA$. Total number of 1.s. parameters $=200 . R 1=$ 0.0349 for $1436 F_{\mathrm{o}}>4 \sigma\left(F_{\mathrm{o}}\right)$ and 0.0406 for all 1589 data. $w R 2=0.0815$, $\mathrm{GOF}=1.08$, restrained $\mathrm{GOF}=1.08$ for all data. An ORTEP drawing of compound $\mathbf{3 3}$ with $50 \%$ ellipsoidal probability is shown in Figure 6.

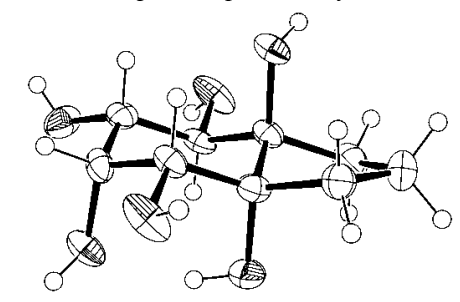

Figure 6. ORTEP drawing for $\mathbf{3 3}$.

Compound 34: A solution of diene $6(200 \mathrm{mg}, 0.847 \mathrm{mmol})$ in acetone/ water $(4: 1,5 \mathrm{~mL})$ was treated with $\mathrm{OsO}_{4}(2 \mathrm{mg}, 1 \mathrm{~mol} \%)$ and NMMO $(200 \mu \mathrm{L}, 0.847 \mathrm{mmol})$, and the resulting mixture was stirred at $0{ }^{\circ} \mathrm{C}$ for $2 \mathrm{~d}$. The reaction was quenched by the addition of solid $\mathrm{NaHSO}_{3}$. After dilution with ethyl acetate $(25 \mathrm{~mL})$, the reaction mixture was filtered through a Celite pad and the filtrate was concentrated under reduced pressure to yield a solid residue. Column chromatography over silica gel (elution with $50 \%$ ethyl acetate/hexane) furnished the diol diacetate $\mathbf{3 4}$ (109 $\mathrm{mg}, 48 \%$ ). M.p. $122.7^{\circ} \mathrm{C}$; IR (KBr): $\tilde{v}=3373,1739 \mathrm{~cm}^{-1} ;{ }^{1} \mathrm{H}$ NMR $\left(300 \mathrm{MHz}, \mathrm{CDCl}_{3}\right)$ : $\delta=6.12(\mathrm{~d}, J=10.2 \mathrm{~Hz}, 1 \mathrm{H}), 5.56(\mathrm{~d}, J=10.2 \mathrm{~Hz}, 1 \mathrm{H}), 5.29(\mathrm{br} \mathrm{s}, 1 \mathrm{H}), 5.24$ (brs, $1 \mathrm{H}), 2.41(\mathrm{~m}, 2 \mathrm{H}), 2.14(\mathrm{~s}, 3 \mathrm{H}), 1.98(\mathrm{~s}, 3 \mathrm{H}), 2.04-1.66(\mathrm{~m}, 4 \mathrm{H})$; ${ }^{13} \mathrm{C}$ NMR $\left(75 \mathrm{MHz}, \mathrm{CDCl}_{3}\right.$ ): $\delta=170.1,169.7,131.9,123.8,89.3,78.2,70.5$, 67.6, 32.6, 26.2, 22.0, 21.1, 18.8; MS (70 eV, EI): $\mathrm{m} / z(\%): 186\left[\mathrm{M}^{+}-\right.$ $2 \mathrm{Ac}+2]$; elemental analysis calcd $(\%)$ for $\mathrm{C}_{13} \mathrm{H}_{18} \mathrm{O}_{6}$ (270.3): $\mathrm{C} 57.77, \mathrm{H}$ 6.71; found: C 57.86, H 6.75 .

Crystal data for the compound 34: Structure was solved by direct methods (SIR92). Refinement was by full-matrix least-squares procedures on $F^{2}$ by using SHELXL-97. Crystal system: monoclinic, space group: $P 2_{1} / n$, cell parameters: $a=7.027(1), b=8.318(1), c=23.002(3) \AA, \beta=92.490(3)^{\circ}, V=$ $1343.26 \AA^{3}, Z=4, \rho_{\text {calcd }}=1.336 \mathrm{~g} \mathrm{~cm}^{-3}, F(000)=576.0, \mu=0.11 \mathrm{~mm}^{-1}, \lambda=$ $0.71073 \AA$. Total number of 1.s. parameters $=244 . \quad R 1=0.0458$ for $1449 F_{\mathrm{o}}>4 \sigma\left(F_{\mathrm{o}}\right)$ and 0.1048 for all 2815 data. $w R 2=0.0994, \mathrm{GOF}=0.820$, restrained $\mathrm{GOF}=0.820$ for all data. An ORTEP drawing of compound $\mathbf{3 4}$ with $50 \%$ ellipsoidal probability is shown in Figure 7.

Compound 35: Diol diacetate $34(100 \mathrm{mg}, 0.370 \mathrm{mmol})$ was hydrolyzed with $\mathrm{K}_{2} \mathrm{CO}_{3}(102 \mathrm{mg}, 0.74 \mathrm{mmol})$ in methanol $(2 \mathrm{~mL})$ followed by purification by chromatography over silica gel $(10 \%$ methanol/ethyl acetate eluent) to give tetrol $35(51 \mathrm{mg}, 75 \%)$. M.p. $146.7^{\circ} \mathrm{C}$; IR (KBr): $\tilde{v}=3364 \mathrm{~cm}^{-1} ;{ }^{1} \mathrm{H}$ NMR $\left(300 \mathrm{MHz}, \mathrm{D}_{2} \mathrm{O}\right): \delta=5.88(\mathrm{~d}, J=10.2 \mathrm{~Hz}, 1 \mathrm{H})$, $5.54(\mathrm{~d}, J=10.2 \mathrm{~Hz}, 1 \mathrm{H}), 4.38(\mathrm{~m}, 1 \mathrm{H}), 4.02(\mathrm{~d}, J=4.8 \mathrm{~Hz}, 1 \mathrm{H}), 2.17-2.07$ $(\mathrm{m}, 1 \mathrm{H}), 1.69-1.67(\mathrm{~m}, 3 \mathrm{H}), 1.53-1.43(\mathrm{~m}, 2 \mathrm{H}) ;{ }^{13} \mathrm{C} \mathrm{NMR}\left(75 \mathrm{MHz}, \mathrm{D}_{2} \mathrm{O}\right)$ : $\delta=130.6,129.9,81.3,80.0,72.3,68.5,33.1,31.219 .0 ; \mathrm{MS}(70 \mathrm{eV}, \mathrm{EI}): \mathrm{m} / \mathrm{z}$

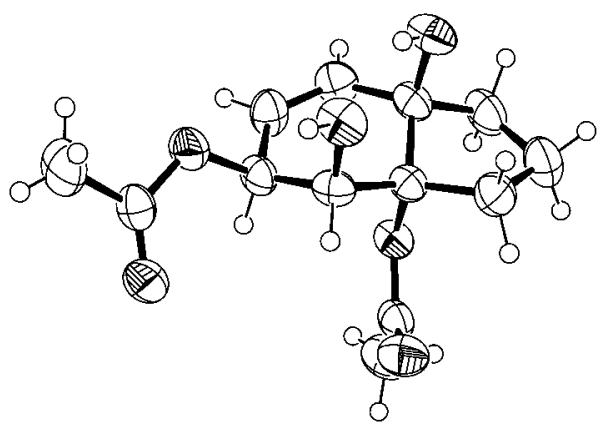

Figure 7. ORTEP drawing for 34.

(\%): $167\left[M^{+}-\mathrm{H}_{2} \mathrm{O}-\mathrm{H}\right]$; elemental analysis calcd (\%) for $\mathrm{C}_{9} \mathrm{H}_{14} \mathrm{O}_{4}$ (186.2): C 58.05, H 7.58; found: C 58.35, H 7.76.

Compound 36: Tetrol 35 ( $45 \mathrm{mg}, 0.242 \mathrm{mmol}$ ) was subjected to a procedure similar to that detailed for the conversion of $\mathbf{1 9}$ to $\mathbf{4}$ but with $\mathrm{OsO}_{4}(2 \mathrm{mg}$, $2 \mathrm{~mol} \%)$ and NMMO $(60 \mu \mathrm{L}, 0.25 \mathrm{mmol})$ in acetone/water $(4: 1,2 \mathrm{~mL})$, followed by purification by chromatography over silica gel $(10 \%$ methanol/ ethyl acetate) to afford $36(33 \mathrm{mg}, 0.152 \mathrm{mmol})$. M.p. $188.6^{\circ} \mathrm{C}$; IR (KBr): $\tilde{v}=3316 \mathrm{~cm}^{-1} ;{ }^{1} \mathrm{H}$ NMR $\left(300 \mathrm{MHz}, \mathrm{D}_{2} \mathrm{O}\right): \delta=4.00(\mathrm{~s}, 2 \mathrm{H}), 3.89(\mathrm{~s}, 2 \mathrm{H})$, 2.17-2.06 (m, 2H), 1.87-1.77 (m, 2H), $1.42-1.34(\mathrm{~m}, 2 \mathrm{H}) ;{ }^{13} \mathrm{C}$ NMR (75 MHz, $\mathrm{D}_{2} \mathrm{O}$ ): $\delta=83.2$ (2C), 74.5 (2C), 69.9 (2C), 31.1 (2C), 19.8; MS (70 eV, EI): $m / z(\%): 166\left[M^{+}-3 \mathrm{H}_{2} \mathrm{O}\right]$.

CCDC 196758 (15), -196759 (18), -196760 (4), -196755 (25), -196756 (26), 196757 (33), -196754 (34) contain the supplementary crystallographic data and can be obtained free of charge via www.ccdc.cam.ac.uk/conts/ retrieving.html (or from the Cambridge Crystallographic Data Centre, 12 Union Road, Cambridge CB2 1EZ, UK; fax: $(+44) 1223-336-033$; or deposit@ccdc.cam.uk).

\section{Acknowledgements}

R.S.S. and M.K.B. would like to thank CSIR (India) and UGC (India), respectively, for the award of a research fellowship. We thank the CCD facility at IISc for help with the X-ray crystal structure determination.

[1] For leading reviews dealing with the chemistry and biology of inositols, see: a) M. J. Berridge, R. F. Irvine, Nature 1984, 312, 315; b) M. J. Berridge, R. F. Irvine, Nature 1989, 341, 197; c) B. V. L. Potter, Nat. Prod. Rep. 1990, 1; d) B. V. L. Potter, D. Lampe, Angew. Chem. 1995, 107, 1933; Angew. Chem. Int. Ed. Engl. 1995, 34, 1933; e) K. Hinterding, D. A. Díaz, H. Waldmann, Angew. Chem. 1998, 110, 716; Angew. Chem. Int. Ed. Engl. 1998, 37, 688; f) Y-T. Chang, G. R. Rosania, S-K. Chung, Exp. Opin. Ther. Patents 2001, 11, 1.

[2] M. J. Berridge, Nature 1993, 361, 315.

[3] For selected references on inositol analogues, see: a) A. M. Riley, B. V. L. Potter, J. Org. Chem. 1995, 60, 4970; b) C. Liu, B. V. L. Potter, J. Org. Chem. 1997, 62, 8335; c) A. M. Riley, B. V. L. Potter, Tetrahedron Lett. 1999, 40, 2213; d) H. Sun, G. B. Reddy, C. George, E. J. Meuillet, M. Berggren, G. Powis, A. P. Kozikowski, Tetrahedron Lett. 2002, 43, 2835; e) D. J. Jenkins, A. M. Riley, B. V. L. Potter, J. Org. Chem. 1996, 61, 7719; f) A. P. Kozikowski, A. H. Fauq, G. Powis, D. C. Melder, J. Am. Chem. Soc. 1990, 112, 4528; g) A. B. Cheikh, L. E. Craine, J. Zemlicka, M. H. Heeg, Carbohydr. Res. 1990, 199, 19; h) A. Schnaars, C. Schultz, Tetrahderon 2001, 57, 519.

[4] For recent synthetic studies on inositols, see: a) Y. U. Kwon, C. Lee, S. K. Chung, J. Org. Chem. 2002, 67, 3327; b) H. Takahashi, H. Kittaka, S. Ikegami, J. Org. Chem. 2001, 66, 2705; c) K. S. Kim, J. I. Park, H. K. Moon, H. Yi, Chem. Commun. 1998, 1945; d) Y. Landias, Chimia 1998 , 52, 104; e) A. M. Riley, D. J. Jenkins, B. V. L. Potter, Carbohydr. Res 1998, 314, 277; f) W. Motherwell, A. S. Williams, Angew. Chem. 1995, 107, 2207; Angew. Chem. Int. Ed. Engl. 1995, 34, 2031; g) T. Hudlicky, M. Mandel, J. Rouden, R. S. Lee, B. Bachmann, T. Dudding, K. J. 
Yost, J. S. Merola, J. Chem. Soc. Perkin Trans. 1 1994, 1553; h) C. Jaramillo, M. M. Lomas, Tetrahedron Lett. 1991, 32, 2501.

[5] a) C. J. Volkmann, G. M Chateauneuf, J. Pradhan, A. T. Baumann, R. E. Brown, P. P. N. Murthy, Tetrahedron Lett. 2002, 43, 4853; b) L. G. Barrientos, P. P. N. Murthy, Carbohydr. Res. 1996, 296, 39; c) C. Liang, C. S. Ewig, T. R. Stouch, A. T. Hagler, J. Am. Chem. Soc. 1994, 116, 3904; d) I. Lee, J. H. Sohn, S. C. Kim, Y. K. Jeon, J. Korean Chem. Soc. 1979, 3, 271.

[6] Cyclohexadiene-trans-diols (trans-CHD), unlike their cis counterparts, are not easily accessible and synthetic access to them is generally tedious (ref. [7a-d]). However, a preparative route to trans-CHD by using recombinant $E$. coli cells has been reported (ref. [7e]). Annulated trans-CHD and its derivatives to the best of our knowledge remain unknown.

[7] a) B. M. Trost, L. S. Chupak, T. Lübbers, J. Am. Chem. Soc. 1998, 120, 1732; b) B. P. McKibben, G. S. Barnosky, T. Hudlicky, Synlett 1995, 806 ; c) D. K. Boyd, N. D. Sharma, H. Dalton, D. A. Clarke, Chem. Commun. 1996, 45; d) H. B. Mereyala, M. Pannala, J. Chem. Soc. Perkin Trans. 1 1997, 1755; e) D. Franke, G. A. Sprenger, M. Müller, Angew. Chem. 2001, 113, 578; Angew. Chem. Int. Ed. Engl. 2001, 40, 555.

[8] a) M. G. Banwell, J. H. Ryan, D. A. Winkler, Aust. J. Chem. 1991, 44, 593; b) W. Hückel, U. Wörffel, Chem. Ber. 1956, 89, 2098.

[9] We regard the annulated cyclohexadiene trans-diols 5 and $\mathbf{6}$ as extremely versatile new building blocks which can be employed to generate a variety of natural product analogues in a manner reminiscent of the use of cis-cyclohexadiene diols derived from benzene derivatives. See: T. Hudlicky, D. Gonzalez, D. T. Gibson, Aldrichimia Acta 1999, 32, 35.

[10] E. Giovannini, H. Wegmüller, Helv. Chim. Acta 1958, 41, 933.

[11] Conduritols in their own right are important biologically active natural products of current interest (see ref. [12]) and our synthetic efforts described here also provide access to a range of novel ringannulated bicyclic conduritols.

[12] a) M. Balci, Pure Appl. Chem. 1997, 69, 97; b) D. C. Billington, F. P. Sierra, S. Beaubras, J. Duhault, J. Episnal, S. Challal, in Carbohydrate Mimics-Concepts and Methods (Ed.: Y. Chapleur), Wiley-VCH, Weinheim, 1998, p. 433.

[13] a) S. J. Angyal, G. J. H. Melrose, J. Chem. Soc. 1965, 6494; b) H. Paulsen, Adv. Carbohydr. Chem. Biochem. 1971, 26, 127; c) V. I. Shvets, Russ. Chem. Rev. 1974, 43, 488.

[14] a) J. K. Cha, W. J. Christ, Y. Kishi, Tetrahedron Lett. 1983, 24, 3943; b) T. J. Donohoe, Synlett 2002, 1233.

[15] The site of annulation on the inositol moiety has been identified by following the corresponding parent inositol numbering. For inositol numbering see, IUPAC-IUB 1973 recommendation for cyclitols: Pure Appl. Chem. 1974, 37, 283 and refs. [1b] and [4b].

[16] G. A. Jeffrey, Y. Yeon, Carbohydr. Res. 1987, 159, 211.

[17] I. N. Rabinowitz, J. Kraut, Acta Crystallogr. 1964, 17, 159. 